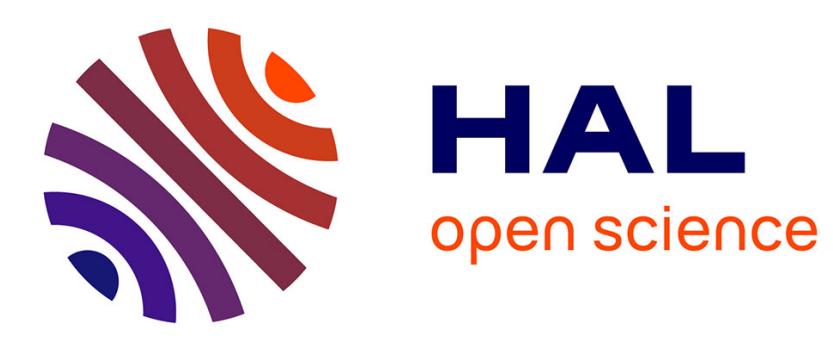

\title{
Theoretical and numerical analysis of the lattice kinetic scheme for complex-flow simulations
}

\author{
S. Jafar Hosseini, N. Darabiha, D. Thevenin
}

\section{To cite this version:}

S. Jafar Hosseini, N. Darabiha, D. Thevenin. Theoretical and numerical analysis of the lattice kinetic scheme for complex-flow simulations. Physical Review E, 2019, 99 (2), 10.1103/PhysRevE.99.023305. hal-02114001

\section{HAL Id: hal-02114001 \\ https://hal.science/hal-02114001}

Submitted on 29 Apr 2019

HAL is a multi-disciplinary open access archive for the deposit and dissemination of scientific research documents, whether they are published or not. The documents may come from teaching and research institutions in France or abroad, or from public or private research centers.
L'archive ouverte pluridisciplinaire HAL, est destinée au dépôt et à la diffusion de documents scientifiques de niveau recherche, publiés ou non, émanant des établissements d'enseignement et de recherche français ou étrangers, des laboratoires publics ou privés. 


\title{
Theoretical and numerical analysis of the lattice kinetic scheme for complex flow simulations
}

\author{
S.A. Hosseini, ${ }^{1,2,3}$ N. Darabiha, ${ }^{2}$ and D. Thévenin ${ }^{1}$ \\ ${ }^{1}$ Laboratory of Fluid Dynamics and Technical Flows, \\ University of Magdeburg "Otto von Guericke", D-39106 Magdeburg, Germany \\ ${ }^{2}$ Laboratoire EM2C, CNRS, CentraleSupélec, \\ Université Paris-Saclay, 3 rue Joliot Curie, \\ 91192, Gif-sur-Yvette Cedex, France \\ ${ }^{3}$ International Max Planck Research School (IMPRS) for Advanced \\ Methods in Process and Systems Engineering, Magdeburg, Germany
}

(Dated: April 29, 2019) 


\begin{abstract}
The lattice kinetic scheme (LKS) is a modified version of the classical single relaxation time lattice Boltzmann method. Although used for many applications, especially when large variations in viscosity are involved, a thorough analysis of the scheme has not been provided yet. In the context of this work, the macroscopic behavior of this scheme is evaluated through the ChapmanEnskog analysis. It is shown that the additional degree of freedom provided in the scheme allows for an independent control of higher-order moments. These results are further corroborated by numerical simulations. The behavior of this numerical scheme is studied for selected external and internal flows to clarify the effect of the free parameter on the different moments of the distribution function. It is shown that it is more stable than SRT (single relaxation time) when confronted to fully periodic under-resolved simulations (especially for $\lambda \approx 1$ ). It can also help minimize the error coming from the viscosity-dependence of the wall position when combined with the bounce-back approach; although still present, viscosity-dependence of the wall position is reduced. Furthermore, as shown through the multi-scale analysis, specific choices of the free parameter can cancel out the leading-order error. Overall the LKS is shown to be a useful and efficient alternative to the SRT method for simulating numerically complex flows.
\end{abstract}

PACS numbers: 47.11.-j

Keywords: lattice Boltzmann; lattice kinetic scheme; turbulent flows; computational fluid dynamics 


\section{INTRODUCTION}

During the past couple of decades, the lattice Boltzmann (LB) method has emerged as an alternative class of numerical solvers for the classical conservation equations describing fluid flow, most prominently the Navier-Stokes (NS) equations complemented by conservation of mass and energy. It has been used in a wide range of application areas, from single-phase turbulent flows to multi-phase ${ }^{1}$ or multi-species flows ${ }^{2}$. As indicated by its name, LB is a solver for a truncated, discretized -in phase-space- form of the Boltzmann transport equation with the Bhatnagar-Gross-Krook (BGK) approximation for the collision operator. The set of discrete particle velocities (also called stencil) is defined with regard to a number of restrictions on the low-order moments, needed to recover the correct conservation equations in the hydrodynamic limit. It can be formally derived through a projection of the distribution function onto a basis of Hermite polynomials followed by a Gauss-Hermite quadrature ${ }^{8}$. The space and time-discretized form of the scheme results in a rather simple, so-called "Stream and Collide" algorithm. The simplicity of the algorithm, along with the local nature of all involved discrete operators, are major assets as compared to other NS-based incompressible solvers. The classical LB scheme is not an incompressible solver per se; it rather corresponds to a low-Mach number isothermal compressible solver. While the Poisson equation used in classical incompressible solvers enforces a divergence-free velocity field, and therefore infinite sound speed, LB shows a finite sound-speed. The compressible equation of state in LB is the main reason for its locality. Apart from the algorithm itself, the ease of implementation of many boundary conditions - especially concerning walls- is another major advantage of LB. The simple Bounce-Back (BB) boundary condition and all its derivatives, commonly used to model static and moving walls, are one appealing aspect of the LB scheme.

All of the previously mentioned properties make the LB method an ideal candidate for direct numerical simulation of complex flows. But to be able to safely use this tool, one must be aware of the numerical artifacts associated with the approach and know how to identify their effects and minimize or - possibly- fully eliminate them.

The standard LB approach, called Single Relaxation Time method (SRT) shows a rather narrow area of stability and applicability. For example, for flows involving large variations in viscosity -leading to large relaxation coefficients in some areas, Burnett and super-Burnett scale dynamics ${ }^{9}$ become dominant over NS terms, leading to incorrect results. On the other 
end of the spectrum, for under-resolved high Reynolds flows, the SRT scheme rapidly becomes unstable. The past twenty years have witnessed the emergence of a variety of solutions to this issue. Most notably, dissipation control through the concept of an entropy functional (Entropic Lattice Boltzmann Method) ${ }^{10} 13$, regularization of the second-order moment of the distribution function (Regularized Lattice Boltzmann Method) ${ }^{14}$, application of the collision operator in moments space (Mutliple Relaxation Time Lattice Boltzmann), application of the collision operator in a frame moving at the local speed of the fluid through the concept of central moments (Central Moments and Cascaded Lattice Boltzmann Method) $15-17$, or other approaches such as the Highly Stable Lattice Boltzmann Method 18 . Additionally, the BB boundary condition, although straightforward and advantageous in terms of numerical properties, comes with a number of issues. One of the well-known issues associated with the BB boundary condition is that the actual position of the "Wall" is viscosity-dependent. For the wall to be located exactly half-way between the last fluid node and its neighboring solid point, the non-dimensional viscosity must be $\nu=1 /(4 \sqrt{3})^{19}$. For smaller values of the non-dimensional viscosity, the position of the wall will get closer to the last fluid node, and in the worst case results in a discrepancy of $\delta x / 2$ in the position of the wall for $\nu=0$. Conversely, for larger non-dimensional viscosities, the effective position of the wall will get farther away from the fluid node -beyond the neighboring solid node. This limits applicability of the numerical scheme to a rather narrow range of time steps and grid sizes. To overcome this issue, one approach is to employ other boundary conditions, such as the non-equilibrium $\mathrm{BB}^{20}$ or non-equilibrium extrapolation ${ }^{21}$ methods. Another approach, widely used in the literature, is to apply the collision operator in momentum space instead of phase-space ${ }^{22123}$, resulting in additional degrees of freedom -the individual relaxation coefficients of the chosen moments. This approach, called the Multiple Relaxation Time method (MRT) to differentiate it from SRT, gives the user additional degrees of freedom to adjust the bulk viscosity coefficient and relaxation coefficients of other higher-order moments. It can be shown ${ }^{19}$ that setting the relaxation rate, $s_{q}$, of the odd-order moments to:

$$
s_{q}=8 \frac{2 / \tau-1}{8 / \tau-1}
$$

as function of the relaxation coefficient $\tau$ places the wall half-way between the solid and fluid nodes regardless of the non-dimensional viscosity value.

Methods based on the application of relaxation in momentum space have been shown to 
be an efficient remedy to the previously mentioned issue and instabilities for under-resolved simulations - for non-dimensional viscosities close to zero. However they come with problems of their own. In particular, MRT leads to higher computational cost and a more complex algorithm. Additionally, universally-accepted directives are still missing regarding the additional tuning parameters and degrees of freedom, for instance concerning the choice of the momentum basis and of the individual relaxation rates. The two-relaxation time method (TRT) is a subset of the MRT scheme where odd-order moments relax at a rate $\tau^{-}$ while even-order ones relax at $\tau^{+19|24| 25}$. This scheme has the advantage of having only one tunable parameter - the even-order relaxation being defined by the physical viscosity; additionally, it is considerably simpler to implement and faster in terms of computational time compared to MRT. Furthermore, it has been shown that setting the odd-order relaxation coefficient to certain "Magic" values, specific goals can be achieved. For example, setting $\left(\tau^{-}-\frac{1}{2}\right)\left(\tau^{+}-\frac{1}{2}\right)$ to $\frac{3}{16}$ makes the wall position viscosity-independent, while setting it to $\frac{1}{12}$ and $\frac{1}{6}$ respectively removes the third-order advection and fourth-order diffusion errors. Considering these potentially promising features, TRT will be involved in the further comparisons for wall-bounded Stokes flows.

The so-called Lattice Kinetic Scheme $(\mathrm{LKS})^{26}+28$ is a modified version of the original SRT approach where the relaxation term is split into two parts: 1) relaxation, with a coefficient not directly proportional to physical dissipation rate, and thus available as free parameter, and 2) an additional convection term computed from the non-equilibrium part of the secondorder moment of the distribution function. This approach has been applied to different areas in recent years, e.g. for non-Newtonian flows, or heat and species transport $\frac{429130}{\text {. A linear }}$ Von-Neumann stability analysis of the scheme for the advection-diffusion equation was also presented in 31 . Although used for many studies, this scheme has not been investigated in detail at the fundamental level. Therefore, the effect of the splitting is not clearly known yet.

To better understand its numerical behavior, in what follows the LKS will be investigated both theoretically and numerically, by comparison with the well-established SRT and TRT models. Theoretical analyses relying on a high-order Chapman-Enskog (CE) development will establish the effect of the modified collision operator on higher-order hydrodynamics, first in the bulk flow, then at boundary nodes. These results will then be further extended and corroborated using a number of numerical test-cases of increasing complexity. 


\section{NUMERICAL METHODS}

Three different numerical formulations are used in the comparative numerical study: classical single relaxation-time (SRT), two-relaxation time (TRT) and lattice kinetic scheme (LKS). For the forcing terms, as detailed later on, Guo's approach is used ${ }^{32}$. It is also worth mentioning that all wall boundaries are modeled through the half-way BB method - unless stated otherwise- without any curved boundary treatment. All of the models and simulations performed in the context of the present study were implemented in and carried out with our in-house lattice Boltzmann solver, ALBORZ (see for instance ${ }^{1415}$ ). All reported simulation results are based on the D2Q9 (for 2-D) and D3Q19 (for 3-D) stencils.

\section{A. Single relaxation time}

The SRT method consists of a set of probability distribution functions for particles moving with a given set of velocity vectors constrained by the stencil, evolving in time through the following equation 33 :

$$
\begin{aligned}
f_{\alpha}\left(\mathbf{x}+\mathbf{c}_{\alpha} \delta t, t+\delta t\right)-f_{\alpha}(\mathbf{x}, t)= & \\
& -\frac{1}{\tau}\left[f_{\alpha}(\mathbf{x}, t)-f_{\alpha}^{(e q)}(\mathbf{x}, t)\right]+F_{\alpha}(\mathbf{x}, t)
\end{aligned}
$$

where $\tau$ is the relaxation coefficient and $F_{\alpha}(\mathbf{x}, t)$ the external force term detailed in subsection II D. The equilibrium distribution function, $f_{\alpha}^{(e q)}$ is defined as:

$$
f_{\alpha}^{(e q)}(\mathbf{x}, t)=w_{\alpha} \rho\left[1+\frac{\mathbf{c}_{\alpha} \cdot \mathbf{u}}{c_{s}^{2}}+\frac{\mathbf{u} \otimes \mathbf{u}:\left(\mathbf{c}_{\alpha} \otimes \mathbf{c}_{\alpha}-c_{s}^{2} \mathbb{I}\right)}{2 c_{s}^{4}}\right]
$$

where $c_{s}$ is the lattice speed of sound and $\mathbb{I}$ is the identity matrix. The Kronecker outer product is represented by " $\otimes "$ while ":" designates the Frobenius inner product. The relaxation coefficient $\tau$ is given by the following equation:

$$
\nu=c_{s}^{2} \delta t\left(\tau-\frac{1}{2}\right)
$$

This system of equation is shown to recover the NS system of equations up to second-order. A multi-scale analysis of the SRT model is given in Appendix A. 


\section{B. Two-relaxation time}

The TRT scheme is a subset of the more general MRT model where even and odd-order moments relax at two different rates. The discrete time-evolution equation is defined as ${ }^{33}$ :

$$
\begin{aligned}
f_{\alpha}\left(\mathbf{x}+\mathbf{c}_{\alpha} \delta t, t+\delta t\right)-f_{\alpha}(\mathbf{x}, t) & = \\
-\frac{1}{\tau^{+}}\left[f_{\alpha}^{+}(\mathbf{x}, t)-\right. & \left.f_{\alpha}^{(e q)^{+}}(\mathbf{x}, t)\right] \\
& -\frac{1}{\tau^{-}}\left[f_{\alpha}^{-}(\mathbf{x}, t)-f_{\alpha}^{(e q)^{-}}(\mathbf{x}, t)\right]+F_{\alpha}(\mathbf{x}, t),
\end{aligned}
$$

where $f_{\alpha}^{(e q)^{-}}, f_{\alpha}^{(e q)^{+}}, f_{\alpha}^{-}$and $f_{\alpha}^{+}$are defined as:

$$
\begin{aligned}
f_{\alpha}^{(e q)^{-}} & =\frac{f_{\alpha}^{(e q)}-f_{\bar{\alpha}}^{(e q)}}{2}, \\
f_{\alpha}^{(e q)^{+}} & =\frac{f_{\alpha}^{(e q)}+f_{\bar{\alpha}}^{(e q)}}{2}, \\
f_{\alpha}{ }^{-} & =\frac{f_{\alpha}-f_{\bar{\alpha}}}{2}, \\
f_{\alpha}{ }^{+} & =\frac{f_{\alpha}+f_{\bar{\alpha}}}{2} .
\end{aligned}
$$

While $\tau^{-}$is a free parameter, $\tau^{+}$is fixed by the viscosity just as in Eq. 4 , and $\bar{\alpha}$ is the direction opposite $\alpha$. For the TRT method, the half-way bounce-back boundary condition places the wall exactly half-way under the following condition ${ }^{33}$ :

$$
\frac{4}{3}\left(\tau^{+}-\frac{1}{2}\right)\left(\tau^{-}-\frac{1}{2}\right)=\frac{1}{4} .
$$

Thus, the possibility to freely tune one of the relaxation parameters (at the difference of the SRT method) gives the user the possibility to make the wall position viscosity-independent.

\section{Lattice kinetic scheme}

This approach is essentially a modified version of the classical SRT model, initially developed for flows involving large variations of the relaxation coefficient. It has been applied to both classical flow solvers and LB solvers for passive scalars such as temperature and concentration. It consists of a time-evolution equation similar to that of the SRT ${ }^{28}$ :

$$
\begin{array}{r}
f_{\alpha}\left(\mathbf{x}+\mathbf{c}_{\alpha} \delta t, t+\delta t\right)-f_{\alpha}(\mathbf{x}, t)= \\
-\frac{1}{\lambda}\left[f_{\alpha}(\mathbf{x}, t)-f_{\alpha}^{(e q)}(\mathbf{x}, t)\right]+F_{\alpha}(\mathbf{x}, t),
\end{array}
$$


where $\lambda$ is the relaxation coefficient, different from the one appearing in SRT. The equilibrium distribution function is defined as:

$$
\begin{aligned}
f_{\alpha}^{(e q)}(\mathbf{x}, t)=w_{\alpha} \rho \overbrace{\left[1+\frac{\mathbf{c}_{\alpha} \cdot \mathbf{u}}{c_{s}^{2}}+\frac{\mathbf{u} \otimes \mathbf{u}:\left(\mathbf{c}_{\alpha} \otimes \mathbf{c}_{\alpha}-c_{s}^{2} \mathbb{I}\right)}{2 c_{s}^{4}}\right.}^{f_{\alpha}^{(e q, 0)}} & +\underbrace{\left.\frac{A \mathbb{P}(n e q):\left(\mathbf{c}_{\alpha} \otimes \mathbf{c}_{\alpha}-c_{s}^{2} \mathbb{I}\right)}{2 \rho(A-\lambda) c_{s}^{4}}\right]}_{f_{\alpha}^{(e q, 1)}},
\end{aligned}
$$

where $\mathbb{P}^{(n e q)}$ is the non-equilibrium part of the second-order moment tensor of the distribution function. This tensor can be computed as:

$$
\mathbb{P}^{(n e q)}=\sum_{\alpha} \mathbf{c}_{\alpha} \otimes \mathbf{c}_{\alpha}\left[f_{\alpha}-f_{\alpha}^{(e q, 0)}\right]
$$

where the constant $A, \lambda$ and the relaxation coefficient $\tau$ appearing in Eq. 4, are related as:

$$
\nu=c_{s}^{2} \delta t\left(\lambda-A-\frac{1}{2}\right)=c_{s}^{2} \delta t\left(\tau-\frac{1}{2}\right) .
$$

Just as for the SRT model, this set of parameters recovers the NS equations as a compressible conservation equation at macroscopic scale up to second order. Detailed multi-scale analysis of the SRT and LKS models are presented in Appendices A through C. In particular, Appendix B presents the analysis of the LKS model up to second order. The resulting code structure is quite similar to SRT, with an additional step where the components of the second-order moment tensor are computed.

\section{Forcing scheme}

External body forces can be applied through a variety of approaches ${ }^{34}$. The effect of forcing schemes on flow simulations has been discussed in many published articles, e.g. In this work Guo's method will be used ${ }^{32}$. For the SRT scheme the force term is defined as:

$$
F_{\alpha}=w_{\alpha} \rho\left(1-\frac{1}{2 \tau}\right)\left(\frac{\mathbf{c}_{\alpha}}{c_{s}^{2}}+\frac{\mathbf{c}_{\alpha} \otimes \mathbf{c}_{\alpha}-c_{s}^{2} \mathbb{I}}{2 c_{s}^{4}} \cdot \mathbf{u}\right) \cdot \mathbf{F},
$$

where $\mathbf{u}$ is now defined as:

$$
\mathbf{u}=\frac{1}{2} F_{\alpha}+\frac{1}{\rho} \sum_{\alpha} \mathbf{c}_{\alpha} f_{\alpha}
$$


In the context of the TRT and LKS models, Guo's forcing term is respectively applied as $29 \mid 36$ :

$$
\begin{aligned}
& F_{\alpha}=\overbrace{\frac{w_{\alpha} \rho}{c_{s}^{2}}\left(1-\frac{1}{2 \tau^{-}}\right) \mathbf{c}_{\alpha} \cdot \mathbf{F}}^{F_{\alpha}^{-}}+\underbrace{w_{\alpha} \rho\left(1-\frac{1}{2 \tau^{+}}\right)\left(\frac{\mathbf{c}_{\alpha} \otimes \mathbf{c}_{\alpha}-c_{s}^{2} \mathbb{I}}{2 c_{s}^{4}} \cdot \mathbf{u}\right) \cdot \mathbf{F}}_{F_{\alpha}^{+}}, \\
& F_{\alpha}=w_{\alpha} \rho\left(1-\frac{1}{2 \lambda}\right)\left(\frac{\mathbf{c}_{\alpha}}{c_{s}^{2}}+\frac{\mathbf{c}_{\alpha} \otimes \mathbf{c}_{\alpha}-c_{s}^{2} \mathbb{I}}{2 c_{s}^{4}} \cdot \mathbf{u}\right) \cdot \mathbf{F} .
\end{aligned}
$$

\section{EXTERNAL FLOWS}

A multi-scale analysis of both SRT and LKS shows that, although recovering exactly the same macroscopic equations up to second-order, their higher-order hydrodynamics differ: the reader is referred to Appendices A to C. While in the SRT method, higher-order hydrodynamics are dictated by the non-dimensional viscosity, see Eqs. A14 and A15, the LKS comes with a free parameter able to control these moments intervening at the so-called Burnett and super-Burnett scales.

The coefficients appear in the form of polynomial functions of the relaxation coefficient in front of these higher-order moments for the SRT scheme; for relaxation coefficients well-

above 1 , they can dominate over the NS scale dynamics, e.g. $\tau^{2}-\tau+\frac{1}{6}$ at scale $\epsilon^{3}$ as shown in Eq. A15. On the other hand, for small values of the relaxation coefficient these polynomial functions take on negative values, contributing to the amplification of sharp gradients and, therefore, leading to numerical instabilities.

The LKS approach provides a degree of control over these higher-order moments. Hence, a comparative study involving LKS will be presented in this section, first for external flows in the absence of any wall.

Concerning the test-cases, the impact of resolution or under-resolution will be first examined for the double periodic shear layer and then through the more complex 3-D Taylor-Green vortex. Then, acoustic wave dissipation in the linear regime will be checked to establish the effect of the modified collision operator on the bulk viscosity. 


\section{A. Under-resolved flow simulation: Doubly periodic shear layer}

The double shear layer is a well-known test-case used in particular to establish the ability of numerical schemes to cope with unresolved flow features. It was for instance used by Brown \& Minion to assess the stability of a number of finite-difference schemes 3738 . This 2-D flow is made up of two longitudinal shear layers located at $y=L / 4$ and $y=3 L / 4$, combined with periodic boundary conditions. Periodicity allows to study the stability properties of the bulk solver without any effects from boundary condition ${ }^{39}$. It has been established that this field should result in the roll-up of the shear layers -due to a Kelvin-Helmholtz instabilityand eventually the generation of two counter-rotating vortices. For many numerical schemes, the under-resolved simulations of this test-case result in additional spurious vortices, ultimately leading to divergence. The velocity field is initialized through the following functions:

$$
\begin{aligned}
& u_{x}=U_{0} \tanh \left[\alpha\left(0.25-\left|\frac{y}{L}-0.5\right|\right)\right], \forall y \\
& u_{y}=\quad U_{0} \delta \sin \left[2 \pi\left(\frac{x}{L}+0.25\right)\right], \quad \forall x
\end{aligned}
$$

where $U_{0}$ is the maximum flow speed, $\alpha$ controls the thickness of the shear layer and $\delta$ determines the amplitude of the perturbation. In the context of this study the maximum velocity in LB units will be set to $0.04, \alpha$ to 80 and $\delta$ to 0.05 . The Reynolds number defined as $\operatorname{Re}=\frac{U_{0} L}{\nu}$ is fixed at $3 \times 10^{4}$ and will be used in each case to determine the non-dimensional

viscosity. The vorticity contours at $t_{c}=\frac{L}{U_{0}}$ will be shown and compared for each case along with the time-evolution of the average kinetic energy and its standard deviation. The testcase is run for two different solvers, SRT and LKS (for different values of $\lambda$ ) using five different resolutions: $32 \times 32,64 \times 64,128 \times 128,256 \times 256$ and $512 \times 512$. The populations are initialized using Grad's approximation ${ }^{12}$ :

$$
\begin{aligned}
f_{\alpha}(\mathbf{u}, 0)=w_{\alpha} \rho\left(1+\frac{\mathbf{u} \cdot \mathbf{c}_{\alpha}}{c_{s}^{2}}\right) & \\
+ & \frac{w_{\alpha}}{2 c_{s}^{4}}\left(\mathbb{P}^{(0)}+\mathbb{P}^{(1)}-\rho c_{s}^{2} \mathbb{I}\right):\left(\mathbf{c}_{\alpha} \otimes \mathbf{c}_{\alpha}-c_{s}^{2} \mathbb{I}\right),
\end{aligned}
$$

where $\mathbb{P}^{(1)}$ is computed as:

$$
\mathbb{P}^{(1)}=-c_{s}^{2} \tau\left(\mathbf{u} \otimes \nabla+\mathbf{u} \otimes \nabla^{T}\right),
$$

and is evaluated through a central difference finite-difference approximation. Looking at the Chapman-Enskog equations, this expression corresponds to a first-order approximation of 
the population, $f_{\alpha} \approx f_{\alpha}^{(0)}+\epsilon f_{\alpha}^{(1)}$, with a leading-order error of $\epsilon^{2} f_{\alpha}^{(2)}$. This fact is important as it leads to non-negligible dissipation at the start of the simulation for low-resolution $\operatorname{cases}^{40}$.

The time-evolution of the average kinetic energy and its standard deviation for three different resolutions, namely $32 \times 32,64 \times 64$ and $128 \times 128$, and for different values of the free parameter $\lambda$ are shown in Fig. 1 and compared to a reference curve corresponding to the result from a SRT simulation at a very fine resolution of $512 \times 512$. As discussed later on in this subsection, the SRT was unstable for the reduced resolutions shown in these figures, so that only the high-resolution SRT results are shown as a reference (solid lines) here. At resolution $128 \times 128$, the average kinetic energy closely follows the reference curve (black solid line) regardless of the choice of $\lambda$; even the evolution of its standard deviation falls on top of the reference curve at early times, and stays very close to it for selected values of $\lambda$. At lower resolutions, $64 \times 64$ and $32 \times 32$, the dissipation rate corresponding to the slope of the average kinetic energy is better approximated by $\lambda=0.93$, followed by $\lambda=0.65$. Furthermore, at the lowest resolution, for $\lambda=0.515$ and 0.53 , a peak in average kinetic energy is observed around $t=t_{c}$. As shown by the insert in Fig. 1 this peak corresponds to the formation of two spurious vortices. This numerical artifact is not observed for the other choices of $\lambda$, nor at higher resolution. These two spurious vortices also explain the sudden rise in standard deviation around $t=3 t_{c}$ observed in Fig. 1. At this time, the spurious vortices start moving towards the main, physical vortical structures (resulting from the shear layer instability), begin to interact and finally merge with them around $t=4 t_{c}$. The merger causes another peak in standard deviation, followed by rapid dissipation. The vorticity contours at $t=t_{c}$ obtained for all five resolutions and using both SRT and LKS are displayed in Fig. 2. As shown in this figure and in agreement with the previous discussion and results shown in Fig. 3, the SRT model only produces stable results at a resolution of $256 \times 256$ and above; while LKS is stable for all resolutions. Furthermore, even at a very coarse resolution of $32 \times 32$, the physical solution delivered by LKS does not deviate much from the reference, high-resolution results; the average kinetic energy differs by less than $2 \%$, and the slope (associated to the dissipation rate) corresponds also closely to the reference solution. 

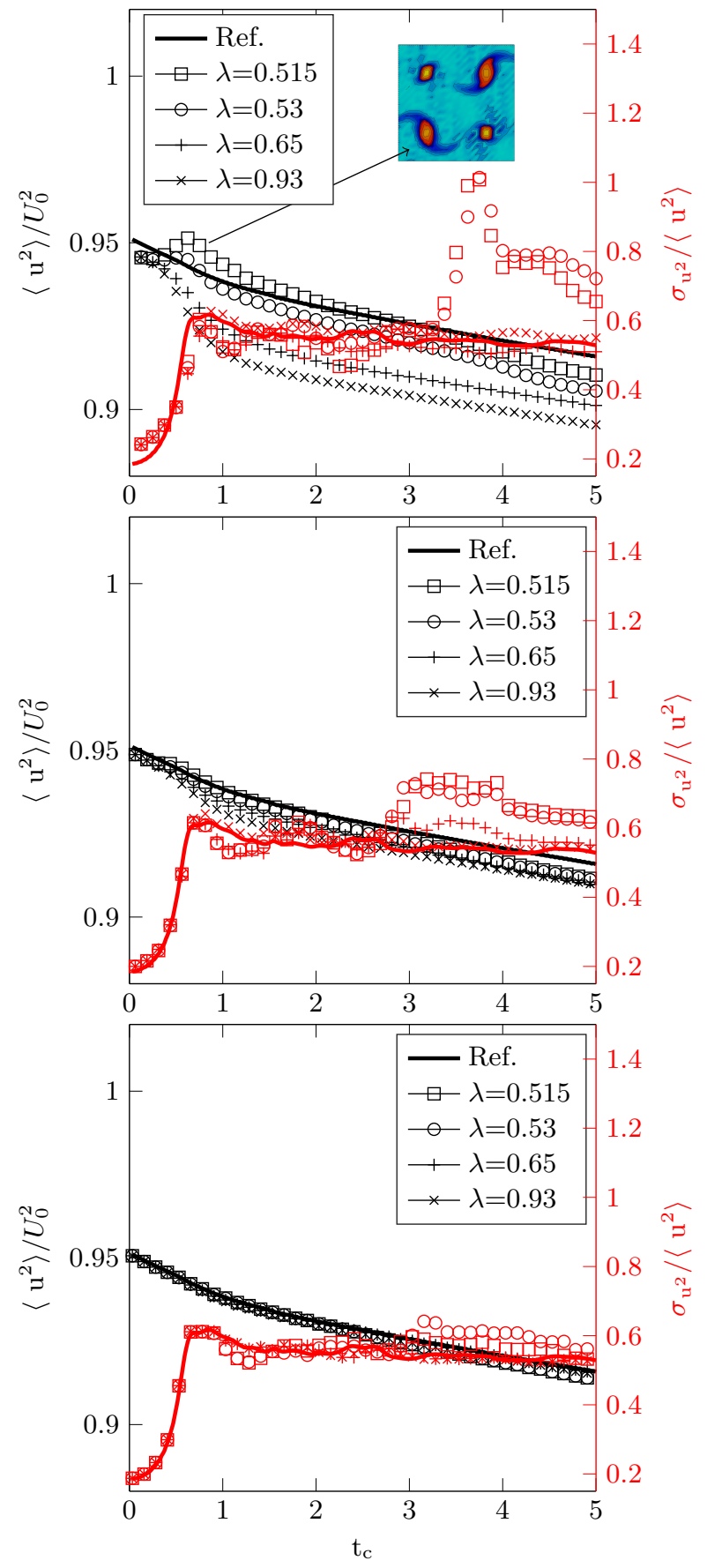

FIG. 1: Evolution of kinetic energy (black) and of its standard deviation (red) for different values of $\lambda$ (see legend) at resolutions (from top to bottom) $32 \times 32,64 \times 64$ and $128 \times 128$

\section{B. 3-D Taylor-Green vortex}

The 3-D Taylor-Green vortex configuration is another fundamental canonical problem to study vortex dynamics and turbulent flow transition. This problem consist of an all-around 


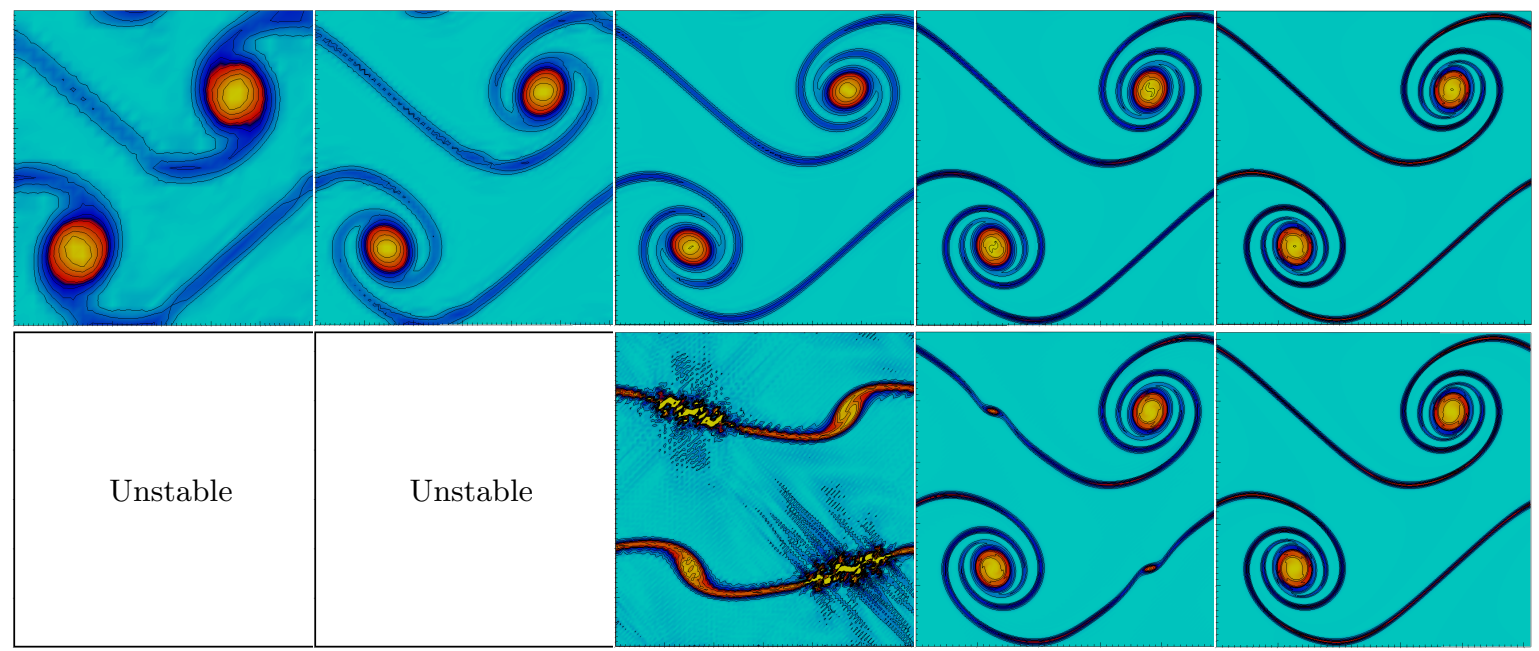

FIG. 2: Doubly periodic shear layer vorticity (magnitude of the z-component) contours at $t=t_{c}$ for SRT (bottom row) and LKS (top row) at five different resolutions, from left to right: $32 \times 32,64 \times 64,128 \times 128,256 \times 256$ and $512 \times 512$. The free parameter $\lambda$ in the LKS is set to 0.93 for these results. The results for the SRT scheme at resolution $128 \times 128$ correspond to $t=0.53 t_{c}$, just before divergence. SRT results cannot be obtained for the lowest resolutions of $32 \times 32$ and $64 \times 64$.
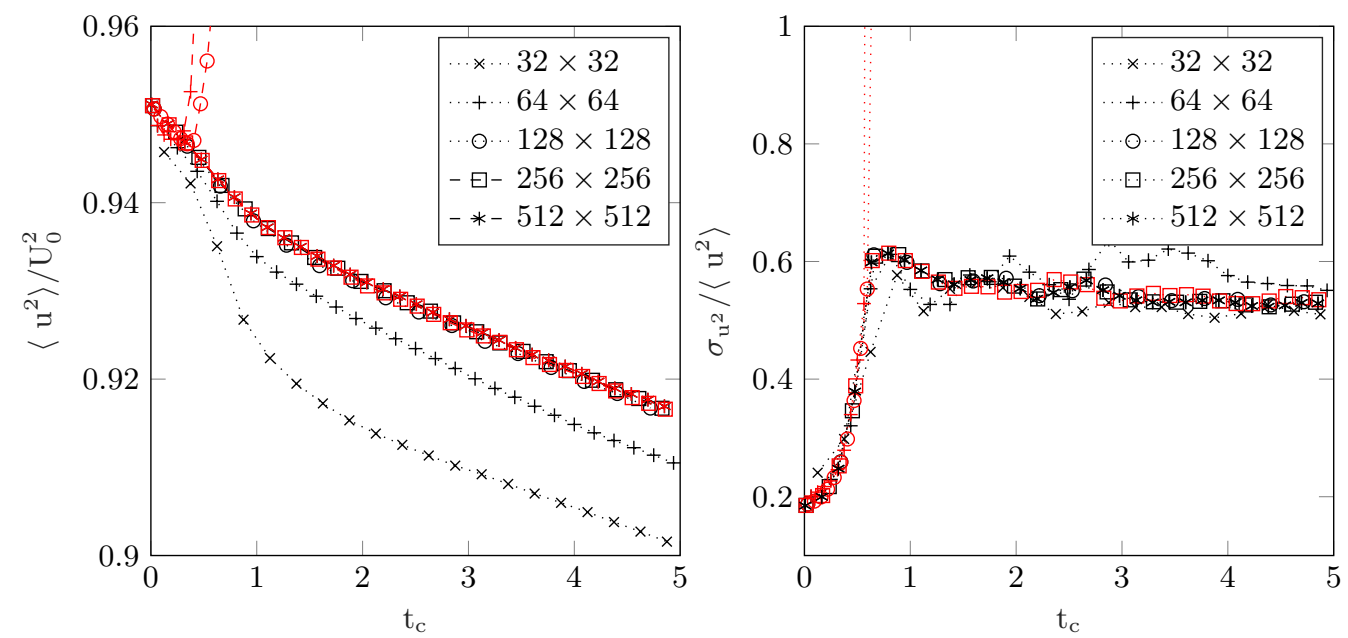

FIG. 3: Evolution of average kinetic energy -left- and of its standard deviation -right, at different resolutions using the SRT (in red) and LKS model with $\lambda=0.93$ (in black). 
periodic cubic simulation domain, initialized using the following equations:

$$
\begin{aligned}
& u_{x}=\quad U_{0} \sin \frac{x}{L} \cos \frac{y}{L} \cos \frac{z}{L}, \\
& u_{y}=\quad-U_{0} \cos \frac{x}{L} \sin \frac{y}{L} \cos \frac{z}{L}, \\
& u_{z}=\quad 0 \text {, } \\
& p=p_{0}+\frac{\rho_{0} U_{0}^{2}}{16}\left(\cos \frac{2 z}{L}+2\right)\left(\cos \frac{2 x}{L}+\cos \frac{2 y}{L}\right),
\end{aligned}
$$

Given that the overall effect of the choice of free parameter has already been established numerically through the previous test-case, all simulations with the LKS in this section will be performed using $\lambda=0.93$. To showcase the stability of the LKS even for 3-D cases, the Taylor-Green vortex at Re=1600 is modeled using both the SRT and LKS approaches with resolutions of: $32^{3}, 64^{3}, 128^{3}$ and $256^{3}$. Additionally a reference simulation at resolution $384^{3}$ is performed using the SRT method. The time-evolution of the average kinetic energy and total enstrophy are shown in Fig. 4, where the total enstrophy -for a compressible flow in $3-\mathrm{D}$ - is computed as:

$$
N^{2}\langle\Omega\rangle=\sum_{x, y, z} \nabla \otimes \mathbf{u}: \nabla \otimes \mathbf{u}
$$

As shown in Fig. 4 the SRT solver is only stable for resolutions above $128^{3}$, while the LKS is stable for all resolutions. At a resolution of $32^{2}$ the SRT blows-up immediately; for a resolution of $64^{3}$ the blow-up happens at $t \approx 10 t_{c}$. Looking at the kinetic energy (Fig. 4 left), it can be seen that the LKS is able to produce acceptable results even at lower resolutions $\left(32^{3}\right.$ and $64^{3}$ ), although at $32^{3}$ visible deviations can be observed. A closer study of the enstrophy evolution (Fig. 4 right) shows that at lower resolutions the simulations are stabilized through the additional higher-order -fourth-order and above- dissipation in LKS model with the chosen value for the free parameter. Being higher-order, this additional numerical dissipation only affects long-wavelength flow features, i.e., eddies not resolved on the grid. This fact is further corroborated by looking at the iso-surfaces of the z-component of the vorticity field, displayed in Fig. 5. As shown there, for under-resolved simulations, larger turbulent structures are unaffected by the higher order additional dissipation terms. The larger structure shown in this figure being resolved down to $32^{3}$, it closely follows the overall structure of its counter-part at higher resolutions. Furthermore, looking at the iso-surfaces obtained for resolutions $128^{3}$ and $256^{3}$, it is observed that the SRT simulation exhibits large wavelength oscillations while the LKS results are smooth. As shown in the theoretical 

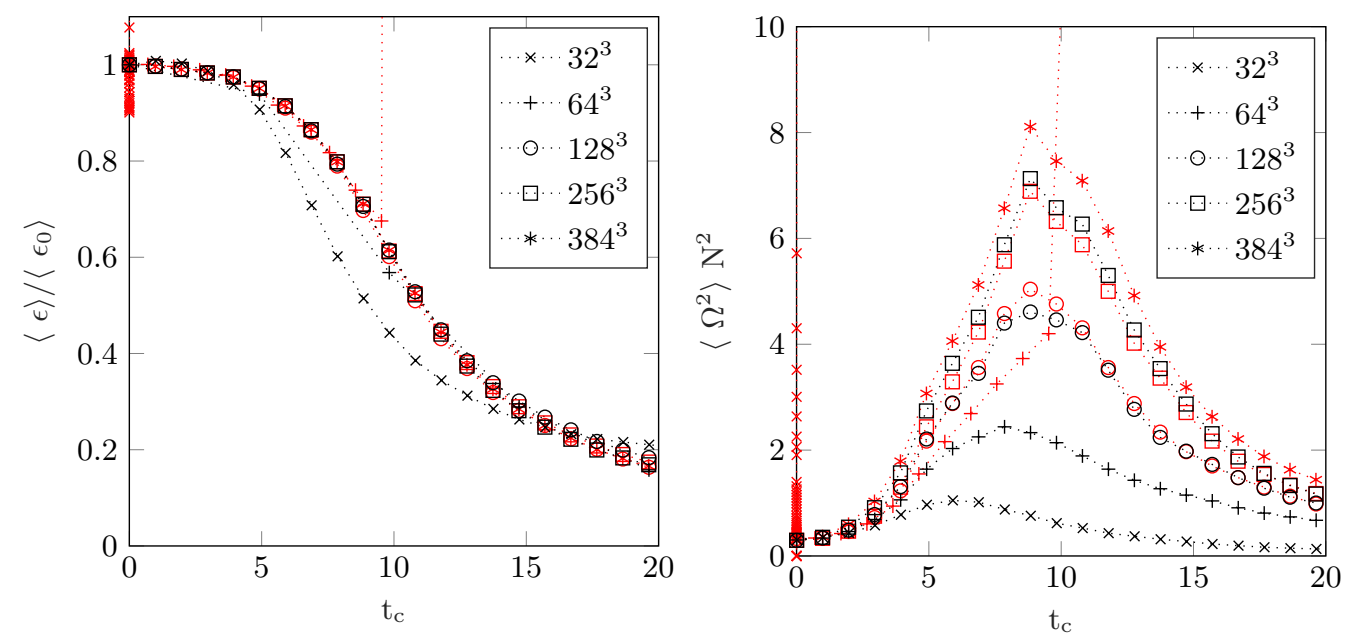

FIG. 4: Evolution of average kinetic energy (left) and enstrophy (right) at different resolutions using the SRT (red) and LKS (black) schemes.
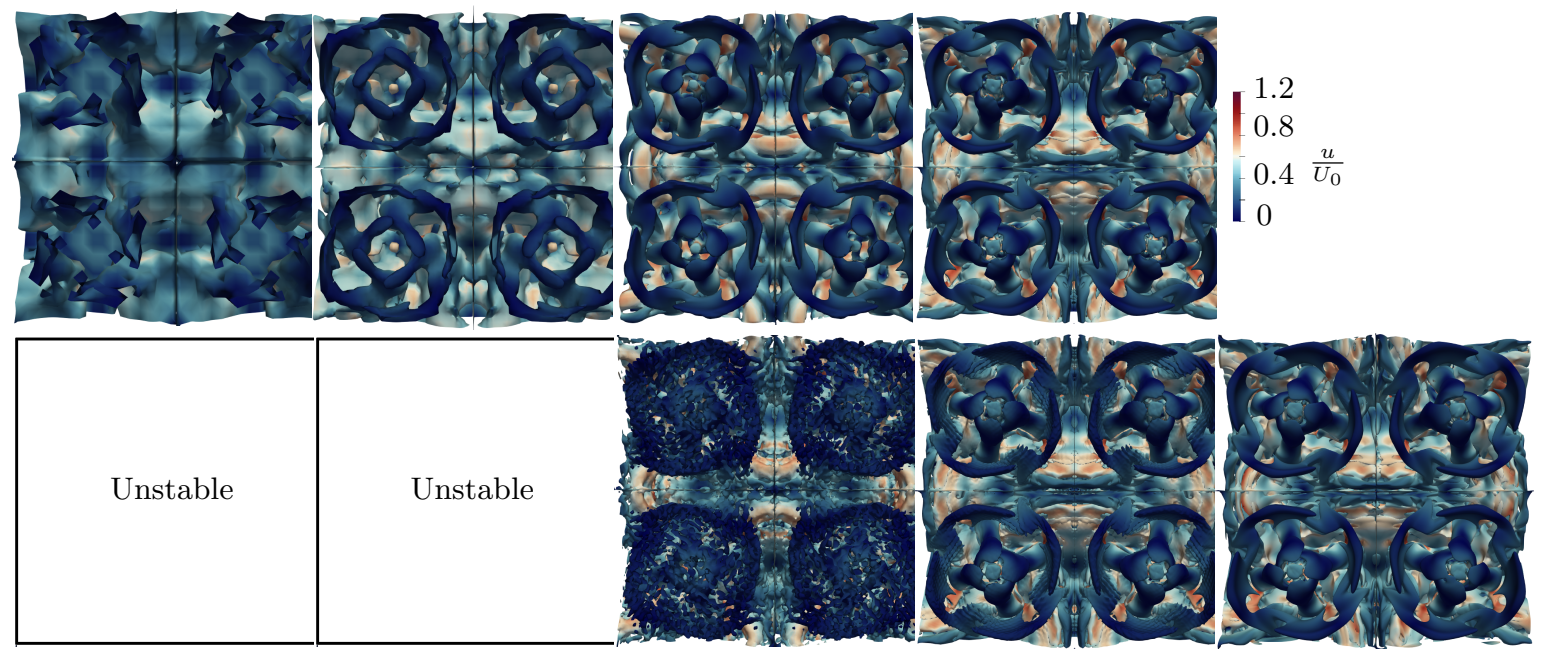

FIG. 5: Iso-surfaces of the $z$-component of vorticity $\omega_{z}$ (bottom view) at $t=10 t_{c}$ obtained using the SRT (bottom) and LKS (top) approaches at resolutions (from left to right) $32^{3}$, $64^{3}, 128^{3}, 256^{3}$ and $384^{3}$

analysis and the previous 2-D test-case (the doubly periodic shear layer), results obtained in this subsection confirm the fact that the LKS free parameter provides a way to control higher-order dispersion and dissipation -independently from the non-dimensional viscosityas a way to either stabilize the simulation or control numerical error. An appropriate choice of this parameter, based on a suitable flow state indicator, could potentially lead to highly stable low-error under-resolved simulations. 


\section{Bulk viscosity and acoustic wave dissipation}

It is a well-known fact that increasing the bulk viscosity can help stabilize under-resolved simulations ${ }^{41}$. Although stable for such simulations, the CE development presented in Appendix $\mathrm{B}$ shows that the LKS does not modify the term $\mathbb{P}^{(1)}$, and therefore does not affect the bulk viscosity. The test-case presented in ${ }^{41}$ is used to numerically establish this fact. In the limit of small velocity and density variations, nonlinear terms in the NS equation can be ignored and acoustic waves can be modeled through the linear theory including losses, which reads for a 3-D flow (only considering flow in the $\mathrm{x}$-direction) 41 :

$$
\partial_{t}^{2} u=c_{s}^{2} \partial_{x}^{2} u+\left(\frac{4}{3} \nu+\eta\right) \partial_{t} \partial_{x}^{2} u
$$

where the bulk viscosity $\eta$ in the context of the classical LB model is fixed at $\frac{2}{3} \nu$. The exact solution of this equation can be shown to be of the form $u \propto e^{i k x+\sigma t}$ where:

$$
\sigma=-\left(\frac{2}{3} \nu+\frac{1}{2} \eta\right) k^{2}+i k c_{s} \sqrt{1-\left(\frac{4}{3} \nu+\eta\right)^{2} \frac{k^{2}}{c_{s}^{2}}}
$$

To evaluate the effective viscosity of the considered numerical schemes, a wave-function is initialized on a $N_{x} \times 2$ domain with periodic boundary conditions as:

$$
\rho=\rho_{0}+\delta \rho \sin \left(\frac{2 \pi x}{N_{x}}\right)
$$

and initial velocity is set to zero. The waves are then left to evolve, and average energy, computed as $E=u^{2}+c_{s}^{2}\left(\rho-\rho_{0}\right)$, is stored for each time-step. Given that in the context of the linear regime, wave decay is an exponential function of the effective viscosity, they are extracted using a linear function interpolation of the Napierian logarithm of energy. In the context of this study, $N_{x}=64, \rho_{0}=1$ and $\delta \rho=10^{-6}$ are used. The effective viscosities are measured for the SRT and LKS models (using different values of $\lambda$ ) over a range of Knudsen numbers computed from Mach and Reynolds numbers, Kn=Ma/Re. The results are displayed in Fig 6 . 


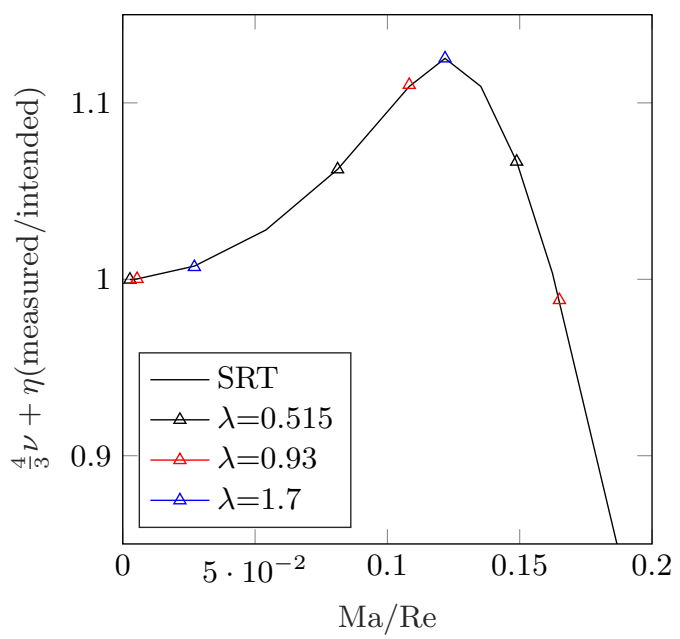

FIG. 6: Effective viscosity for SRT and LKS for three different values of $\lambda(0.515,0.93$ and 1.7), as a function of the Knudsen number $\mathrm{Kn}=\mathrm{Ma} / \mathrm{Rehl}$.

As pointed out in $\frac{41}{}$, by setting $\delta \rho$ to very small values, non-linear terms of order $\mathcal{O}\left(u^{2}\right)$ along with higher-order dissipation terms of order $\mathcal{O}\left(\tau^{2}\right)$ become negligible. Therefore, this figure shows that up to second-order in $\epsilon$, the numerical dissipation (and therefore the bulk viscosity) is not affected by the choice of $\lambda$ in the LKS method.

\section{WALL-BOUNDED FLOWS}

One of the issues of the classical SRT method, when it comes to modeling wall-bounded flows, is that combined with the half-way bounce-back rule it does not always place the wall half-way between the fluid and solid node. In the context of this work, only the half-way bounce-back scheme is considered as it gives us additional insights into the bulk solver (Especially the collision operator). This is mainly because the bounce-back approach operates on specific moments (first order) of the distribution function and the wall position can only be fixed by controlling the relaxation of the first-order moment. It has been shown that the actual position of the wall admits a second-order dependence on the non-dimensional viscosity. The TRT and MRT schemes, with optimal choice of the free parameter, have been shown to be effective remedies to this issue. This is achieved by controlling the relaxation of corresponding moments of the distribution function. To assess the performance of LKS, a 2-D Poiseuille flow is first considered, followed by the evaluation of the permeability of a porous cell with a Stokes flow. 


\section{A. Poiseuille flow}

The 2-D steady Poiseuille flow with a constant body force is an excellent test-case to evaluate the behavior of a second-order numerical solver coupled to wall boundary conditions. Numerical simulations along with analytical study of the SRT solver relying on the half-way bounce-back scheme have shown that the wall position is viscosity-dependent. The velocity at the last fluid node is readily shown to $b e^{33}$ :

$$
u_{0}=u_{x}(f)+\frac{\delta_{x}}{2} \partial_{y} u_{x}(f)+\delta_{x}^{2}\left(1-c_{s}^{2}\right)\left(\tau-\frac{1}{2}\right)^{2} \partial_{y}^{2} u_{x}(f)
$$

where $u_{0}$ and $u_{x}(f)$ are the velocity at the wall and the last fluid node respectively. Associating the previous expression to a second-order Taylor-MacLaurin expansion of velocity at half-way:

$$
u_{0}=u_{x}(f)+\frac{\delta_{x}}{2} \partial_{y} u_{x}(f)+\frac{\delta_{x}^{2}}{8} \partial_{y}^{2} u_{x}(f),
$$

means that for the wall to be placed half-way, the following must always hold:

$$
\left(\tau-\frac{1}{2}\right)^{2}=\frac{1}{8\left(1-c_{s}^{2}\right)}
$$

A multi-scale analysis of the bounce-back rule with LKS, as detailed in Appendix D, results into the following expression:

$$
\left(\tau-\frac{1}{2}\right)=\frac{1}{8\left(\lambda-\frac{1}{2}\right)\left(1-c_{s}^{2}\right)}
$$

According to this result, with the correct choice of the free parameter $\lambda$, the LKS should be able to yield a viscosity-independent and exact solution to the 2-D Poiseuille flow. To confirm this development, the 2-D Poiseuille flow has been modeled using the SRT, TRT and LKS schemes. The error as a function of the non-dimensional viscosity is shown in Fig. 7. The free parameter in the TRT scheme has been set such as to place the wall half-way. For the LKS simulations, a variety of fixed values of $\lambda$ as well as the correlation obtained from Eq. 26 (written simply "LKS" in Fig. 7) have been considered. The spanwise direction is resolved with 10 lattice nodes. As expected, the TRT and LKS schemes with optimal correlations, i.e., when Eqs. 6] and 26 are respectively used to define the free parameter, are error-free (and thus do not appear in the inlay in Fig. 7, being below the error level of $10^{-8}$ ). 


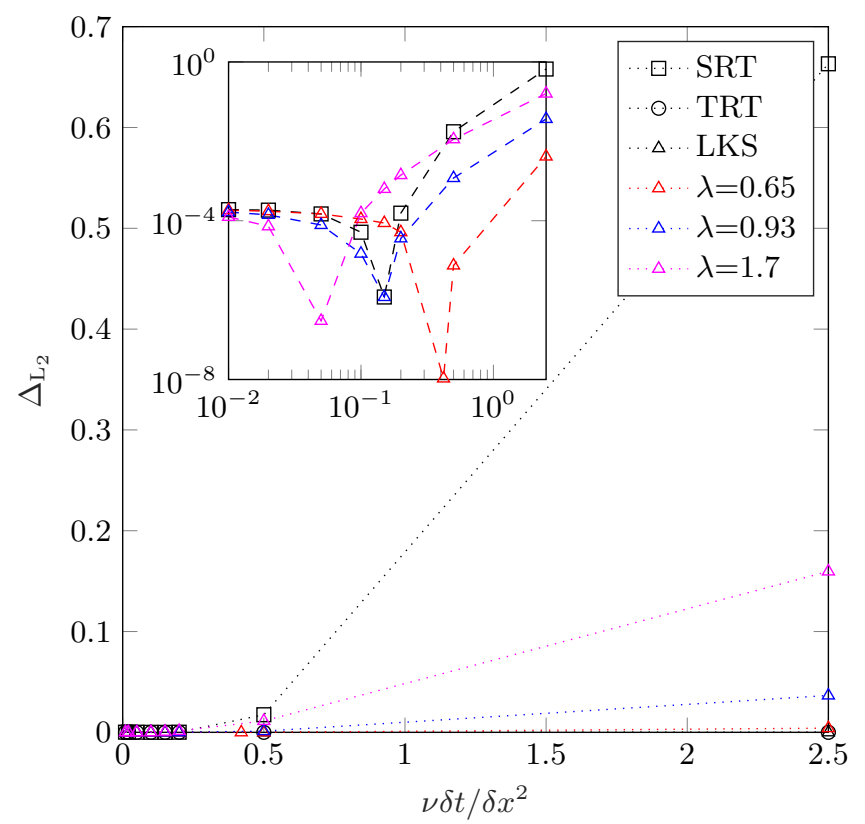

FIG. 7: $L_{2}$-norm of the error associated with the velocity field as a function of the nondimensional viscosity for SRT, TRT with optimal free parameter, LKS with optimal free parameter (simply written "LKS"), and LKS with selected, fixed values of $\lambda$.

On the other hand, the SRT scheme and LKS with fixed values of the free parameter show a noticeable, viscosity-dependent error. The position associated to the minimum error (associated to the position of the wall in the numerical model) shifts to the left for higher values of the free parameter $\lambda$. Using LKS with fixed $\lambda$, although not eliminating the bounceback error, has the advantage of considerably reducing it in comparison with SRT. These results confirm the developments presented in Appendix $\mathrm{D}$. The corresponding validation is only partial, since higher-order effects cannot be assessed for this simple flow with parabolic profile. To go further, the next subsection will focus on the Stokes flow in an exemplary porous cell.

\section{B. Stokes flow in porous media}

In order to evaluate the effect of the non-dimensional viscosity on the simulation, especially effects due to the viscosity-dependence of the position of the wall, a steady-state case was chosen, in which the flow through a simple porous structure is considered. At steady-state and for low Reynolds numbers, non-linear velocity terms being small, the NS 
equations reduce to the Stokes equation. Taking the volume-average of the Stokes equation, it can be shown that the following relation is recovered for a system with a solid phase $\alpha$ and a fluid phase $\beta^{42}$ :

$$
\langle\mathbf{u}\rangle=-\frac{k}{\mu}\left(\nabla \cdot\langle p \mathbb{I}\rangle^{\beta}-\rho \mathbf{F}\right)+k \nabla^{2}\langle\mathbf{u}\rangle^{\beta}
$$

where $\langle\phi\rangle$ is the average value of variable $\phi$ over both phases while $\langle\phi\rangle^{\beta}$ is the average value in phase $\beta$ and $k$ is the permeability. Neglecting the Brinkman correction (last term on the RHS) and body forces, Darcy's equation is recovered $\stackrel{43}{\text { : }}$

$$
\langle\mathbf{u}\rangle=-\frac{k}{\mu} \nabla \cdot\langle p \mathbb{I}\rangle^{\beta}
$$

In the present section, Eq. 28 will be used along with LB simulations of the flow in a periodic domain filled with a body-centered cubic (BCC) solid matrix ${ }^{44}$. The corresponding geometry is shown in Fig. 8. For a cell size $L$, the sphere diameter $D$ is set to $\sqrt{3} L / 2$.

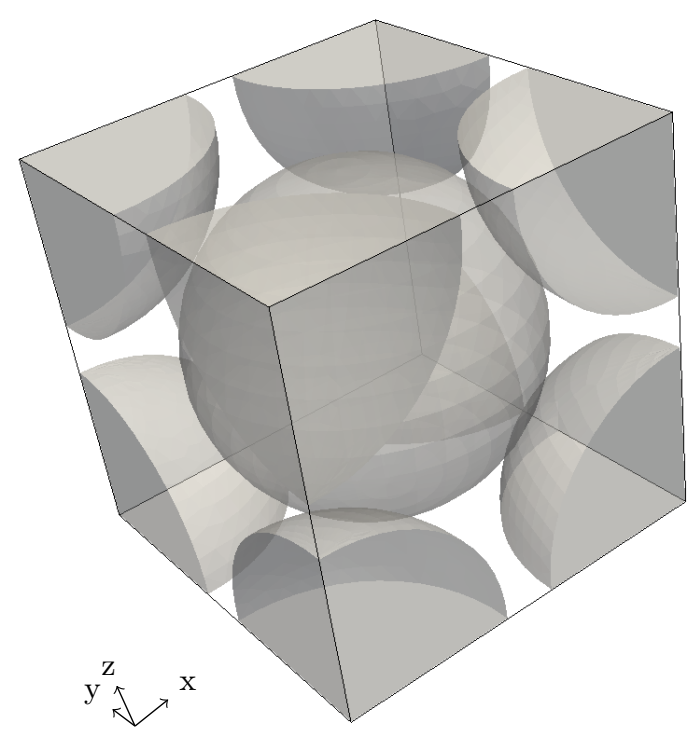

FIG. 8: Body-Centered Cubic cell geometry 


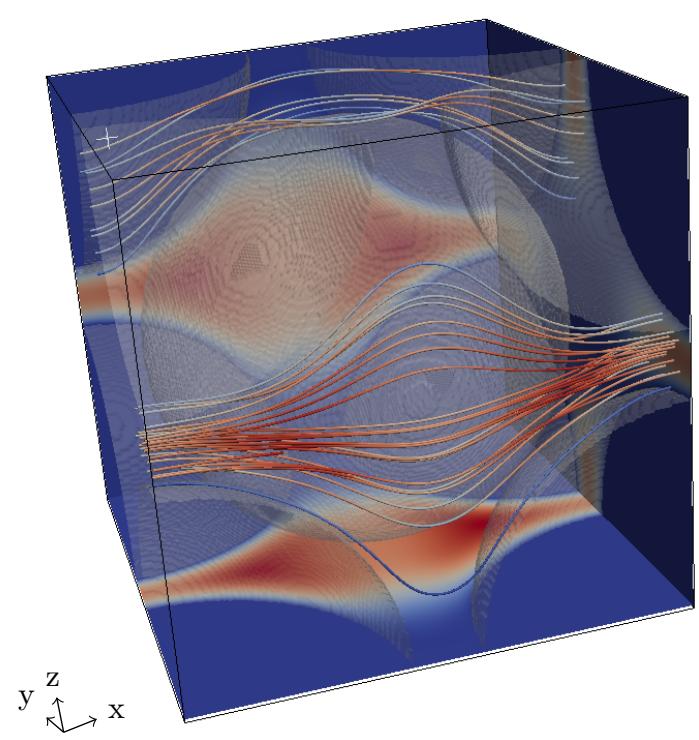

FIG. 9: Steady-state flow -velocity magnitude distribution-obtained in the BCC geometry

In all simulations, periodic boundary conditions are applied in all directions, the flow is initially at rest and with a constant density. It is set in motion by enforcing a constant background pressure gradient over the entire fluid domain. Simulations are stopped once the flow field has reached a given convergence threshold, defined as:

$$
\Delta=\frac{\sum_{i j k}\left|\|\mathbf{u}\|^{t}-\|\mathbf{u}\|^{t-1}\right|}{\sum_{i j k}\|\mathbf{u}\|^{t}} \leq 10^{-6}
$$

Once steady-state has been reached, the velocity is averaged over the simulation domain and the permeability is computed.

The Reynolds number is defined as:

$$
\operatorname{Re}=\frac{\left\langle u_{x}\right\rangle D}{\nu}
$$

where $D$ is the sphere diameter. For the sake of readability, all reported values and simulation parameters will be given in non-dimensional form, using the time-step $\delta t$ and the grid-size $\delta x$. The steady flow structure corresponding to the geometry of Fig. 8 is shown in Fig. 9. First, simulations at very low Reynolds numbers (all below $R e=10^{-2}$ ) are performed for both SRT and the LKS with different values of $\lambda$. Simulations are performed on a $100 \times 100 \times 100$ grid and using the D3Q19 stencil. Since low-Reynolds number flows are considered, anisotropy effects stemming from the defective planes of this stencil are negligible. The non-dimensional viscosity, $\nu \delta t / \delta x^{2}$, will take on values between 0.05 and 
2.4, going both well above and below the value resulting in the wall being located exactly half-way between the fluid and neighboring solid node. For each case, the permeability $k$ is estimated using Eq. 28.

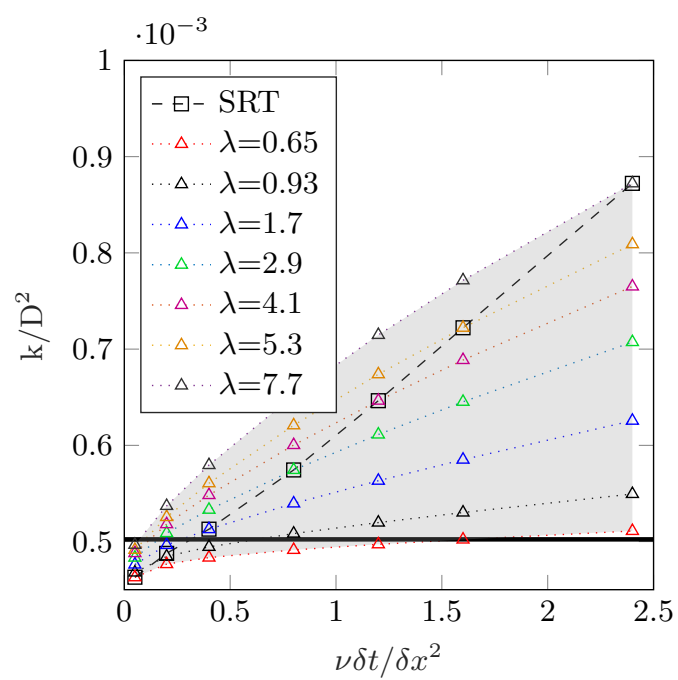

FIG. 10: Effect of the choice of the free parameter $\lambda$ on the viscosity-dependence of permeability for the LKS model. The thick horizontal black line denotes the theoretical solution ${ }^{45}$. The SRT solution is plotted as a dotted black line with square symbols.

It is worth mentioning that for the considered geometry, the porosity $\phi$ is 0.3199 . According to the theoretical study of oscillatory Stokes flows in periodic porous media by Chapman and Higdon ${ }^{45}$, for a BCC cell and for this porosity, the non-dimensional permeability should be $5.023 \times 10^{-4}$. To check the impact of $\lambda$, LKS simulations are performed over a wide range of values for this free parameter. The permeabilities obtained for different values of $\lambda$ are compared in Fig. 10. As shown by this plot, the SRT model exhibits a second-order dependence on the non-dimensional viscosity, going as high as $88 \%$ above the reference non-dimensional permeability. The LKS, on the other hand, shows a more well-behaved evolution. For a fixed value of $\lambda$, the permeability growth is almost linear, at least at higher values of $\nu \delta t / \delta x^{2}$.

Furthermore, small values of $\lambda$ result in a slow growth pace, leading to an acceptable agreement with the reference solution. This observation confirms results discussed previously. To better illustrate this point, results obtained for SRT, LKS with $\lambda$ computed with Eq. 26, and TRT are compared in Fig. 11 . 


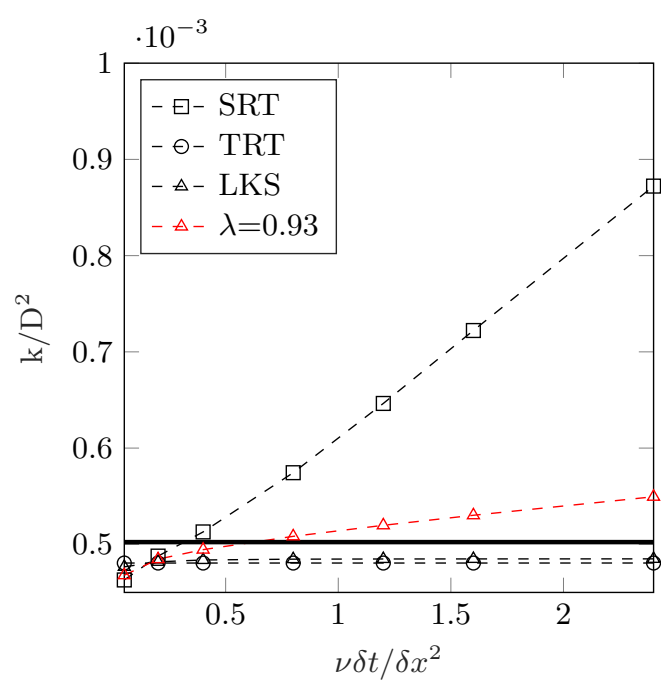

FIG. 11: Viscosity-dependence of permeability for SRT, TRT and LKS. For TRT and LKS, optimal correlations have been used to set the free parameter. The thick horizontal black line denotes the theoretical solution $\frac{45}{4}$.

As shown in this plot, using Eq. 26 to define $\lambda$ results in a permeability which is almost independent from viscosity $\nu \delta t / \delta x^{2}$; this is completely true for large values of the nondimensional viscosity. At smaller values of $\nu \delta t / \delta x^{2}$, although considerably reduced, some slight degree of viscosity-dependence remains. This in turn emphasizes the fact that the splitting performed on the relaxation of the second-order moment of the distribution function does not result in independent control of the relaxation moment affected by the bounce-back boundary condition (first-order moment). For the TRT scheme, as expected the permeability is independent of non-dimensional viscosity. To have a better understanding of the small changes observed at low values of $\nu \delta t / \delta x^{2}$, the simulations have been also performed at two additional resolutions, namely domains of sizes $50 \times 50$ and $25 \times 25$-corresponding to $D=43.3$ and $D=21.65$. The results are plotted in Fig. 12. As shown in this figure, the changes in non-dimensional permeability observed for small viscosities are resolutiondependent. Furthermore, their order in space is higher than two, confirming that Eq. 26 only eliminates viscosity-dependence of the wall position up to second-order in space. It is also worth mentioning that some deviation is observed for the TRT scheme at the lowest resolution, which can be attributed to flow blockage or other geometrical effects caused by the very large discrete voxels. Based on these results, one can see that a correct choice of the free parameter $\lambda$ can lead to an exact positioning of the wall up to second-order. For 


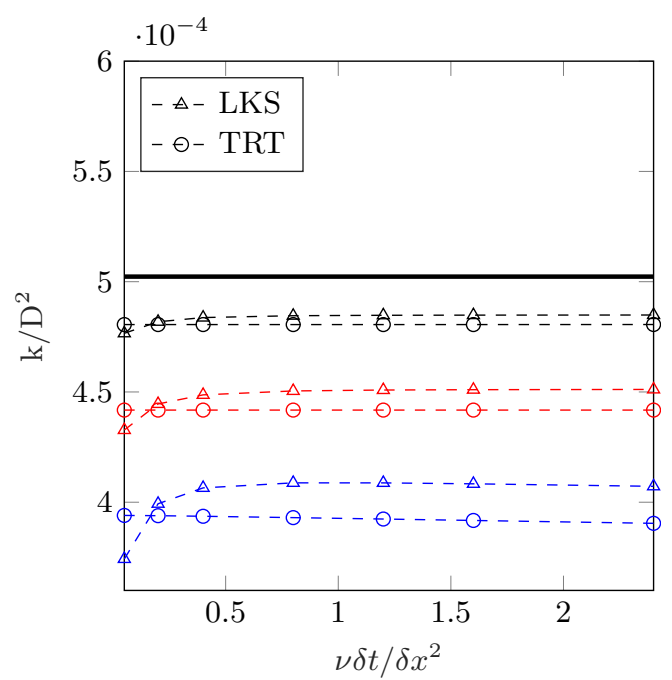

FIG. 12: Non-dimensional permeability as a function of viscosity for TRT and LKS schemes and for three different resolutions: $D=86.6$ (black), $D=43.3$ (red), and $D=21.65$ (blue). The thick horizontal black line denotes the theoretical solution 4 .

general flows, where higher-order derivatives are non-zero and will become important, the position of the wall is not exact. Although LKS with Eq. 26 performs always much better than SRT, other boundary condition schemes (non-equilibrium extrapolation 21, regularized $\underline{46}$ and other moment-based approaches... ) might be better adapted for a correct simulation of wall-bounded complex flows.

\section{CONCLUSIONS AND DISCUSSION}

The LKS is a slightly-modified version of the SRT model, where higher-order moments are controlled by splitting the second-order moment relaxation operator. Previous numerical studies had already established that this approach is very useful and efficient for flows involving large variations in viscosity, such as non-Newtonian flows. The multi-scale analysis presented in this article explains this observation. By splitting the relaxation operator for the second-order moment, the polynomials appearing in front of higher-order moments as function of the relaxation coefficient can be controlled through the free parameter $\lambda$. Furthermore, it has been shown that this control over higher-order moments can allow for more stable simulations of under-resolved bulk flows, while not affecting other important properties such as bulk viscosity. Furthermore, looking at the coefficients appearing in front 
of the higher order moments, $\lambda$ can be defined in a way to cancel out one of them, similar to the TRT method. For example, setting $\lambda=\frac{\tau-1 / 6}{2(\tau-1 / 2)}$, cancels out the second term on the LHS of Eq. C8. However, this choice of free parameter does not result into a stable solver (compared for example to $\lambda=0.93$ ). The choice of $\lambda$ resulting in the most stable solver can be found through a systematic von Neumann analysis of the linearized collision operator. This study is currently being done and will be reported in future publications.

The theoretical analysis along with numerical experiments show that, in combination with the bounce-back boundary condition, the LKS helps fixing properly the position of the wall up to second-order in space. For general flows involving higher-order gradients, although not exact, LKS performs much better than SRT. This choice of $\lambda$, however, does not lead to the most stable simulations (similar to what is observed for the TRT scheme). To be able to perform more realistic wall-bounded flow simulations, other boundary condition schemes such as non-equilibrium extrapolation, regularized etc must be used. Based on the analysis presented in this work, the LKS can be perceived as an alternative to SRT with independent control over higher-order moments. To be able to fully use this scheme for general complex flows and for optimal results two issues have to be resolved: choice of the free parameter, and proper boundary condition schemes not affected by the relaxation coefficient.

\section{ACKNOWLEDGMENTS}

The financial support of the German research foundation (DFG), Germany within the graduate college for "Micro-Macro-Interactions in Structured Media and Particle Systems" (GRK 1554) is gratefully acknowledged. Furthermore S.A.H. would like to thank C. Coreixas and L. Fei for proof reading the article.

1 A. Eshghinejadfard, S. A. Hosseini, and D. Thévenin, AIP Advances 7, 095007 (2017).

2 E. Chiavazzo, I. V. Karlin, A. N. Gorban, and K. Boulouchos, Journal of Statistical Mechanics: Theory and Experiment 2009, P06013 (2009).

3 S. Ayodele, F. Varnik, and D. Raabe, Physical Review E 83, 016702 (2011).

4 S. A. Hosseini, N. Darabiha, and D. Thévenin, Physica A: Statistical Mechanics and its Applications 499, 40 (2018). 
5 S. A. Hosseini, A. Eshghinejadfard, N. Darabiha, and D. Thévenin, Computers \& Mathematics with Applications, doi:10.1016/j.camwa.2017.08.045 (2017).

6 S. Arcidiacono, J. Mantzaras, and I. Karlin, Physical Review E 78, 046711 (2008).

7 N. Prasianakis and K. Boulouchos, International Journal of Modern Physics C 18, 602 (2007).

8 C. Coreixas, G. Wissocq, G. Puigt, J.-F. Boussuge, and P. Sagaut, Physical Review E 96, 033306 (2017).

9 T. Ohwada and K. Xu, Journal of Computational Physics 201, 315 (2004).

10 I. V. Karlin, A. Ferrante, and H. C. Öttinger, EPL (Europhysics Letters) 47, 182 (1999).

11 S. Chikatamarla, S. Ansumali, and I. V. Karlin, Physical review letters 97, 010201 (2006).

12 B. Dorschner, F. Bösch, S. S. Chikatamarla, K. Boulouchos, and I. V. Karlin, Journal of Fluid Mechanics 801, 623 (2016).

13 F. Bösch, S. S. Chikatamarla, and I. V. Karlin, Physical Review E 92, 043309 (2015).

14 J. Latt and B. Chopard, Mathematics and Computers in Simulation 72, 165 (2006).

15 M. Geier, A. Greiner, and J. G. Korvink, Physical Review E 73, 066705 (2006).

16 P. Asinari, Physical Review E 78, 016701 (2008).

17 F. Dubois, T. Fevrier, and B. Graille, Communications in Computational Physics 17, 1088 (2015).

18 Z. Chen, C. Shu, D. Tan, and C. Wu, International Journal for Numerical Methods in Fluids 87, 161 (2018).

19 L.-S. Luo, W. Liao, X. Chen, Y. Peng, and W. Zhang, Physical Review E 83, 056710 (2011).

20 Q. Zou and X. He, Physics of Fluids 9, 1591 (1997).

21 G. Zhao-Li, Z. Chu-Guang, and S. Bao-Chang, Chinese Physics 11, 366 (2002).

22 D. d'Humières, Rarefied Gas Dynamics: Theory and Simulations, Progress in Astronautics and Aeronautics , 450 (1992).

23 D. d'Humières, Philosophical Transactions of the Royal Society of London A: Mathematical, Physical and Engineering Sciences 360, 437 (2002).

24 I. Ginzburg, Advances in Water Resources 28, 1171 (2005).

25 I. Ginzburg and D. d'Humières, Advances in Water Resources 30, 2202 (2007).

26 T. Inamuro, Philosophical Transactions of the Royal Society of London A: Mathematical, Physical and Engineering Sciences 360, 477 (2002). 
27 M. Yoshino, Y.-H. Hotta, T. Hirozane, and M. Endo, Journal of Non-Newtonian Fluid Mechanics 147, 69 (2007).

28 L. Wang, J. Mi, X. Meng, and Z. Guo, Communications in Computational Physics 17, 908 (2015).

29 X. Meng and Z. Guo, Physical Review E 92, 043305 (2015).

30 K. Suzuki and T. Inamuro, International Journal of Modern Physics C 25, 1340017 (2014).

31 S. A. Hosseini, N. Darabiha, D. Thévenin, and A. Eshghinejadfard, International Journal of Modern Physics C 28, 1750141 (2017).

32 Z. Guo, C. Zheng, and B. Shi, Physical Review E 65, 046308 (2002).

33 T. Krüger, H. Kusumaatmaja, A. Kuzmin, O. Shardt, G. Silva, and E. M. Viggen, Springer International Publishing 10, 978 (2017).

34 A. Mohamad and A. Kuzmin, International Journal of Heat and Mass Transfer 53, 990 (2010).

35 L. Fei and K. H. Luo, Phys. Rev. E 96, 053307 (2017).

36 X. Yang, B. Shi, and Z. Chai, Physical Review E 90, 013309 (2014).

37 D. L. Brown and M. L. Minion, Performance of Under-resolved Two-Dimensional Incompressible Flow Simulations, Tech. Rep. (Technical Report Los Alamos National Laboratory, 1995).

38 M. L. Minion and D. L. Brown, Journal of Computational Physics 138, 734 (1997).

39 D. Ricot, S. Marié, P. Sagaut, and C. Bailly, Journal of Computational Physics 228, 4478 (2009).

40 K. K. Mattila, L. A. Hegele Jr, and P. C. Philippi, Physical Review E 91, 063010 (2015).

41 P. J. Dellar, Physical Review E 64, 031203 (2001).

42 S. Whitaker, Transport in porous media 1, 3 (1986).

43 S. Whitaker, The method of volume averaging, Vol. 13 (Springer Science \& Business Media, 2013).

44 A. Eshghinejadfard, L. Daróczy, G. Janiga, and D. Thévenin, International Journal of Heat and Fluid Flow (2016).

45 A. Chapman and J. Higdon, Physics of Fluids A: Fluid Dynamics 4, 2099 (1992).

46 O. Malaspinas, B. Chopard, and J. Latt, Computers \& Fluids 49, 29 (2011).

47 Y.-H. Qian and Y. Zhou, Physical Review E 61, 2103 (2000).

48 F. Dubois and P. Lallemand, Journal of Statistical mechanics: theory and experiment 2009, P06006 (2009). 


\section{Appendix A: Higher-order CE analysis of classical SRT-LBM}

Following standard multi-scale analysis approach, the following expansions are made (the forcing term not being affected by the collision model, it is not considered in the developments):

$$
\begin{gathered}
f_{\alpha}=f_{\alpha}^{(0)}+\epsilon f_{\alpha}^{(1)}+\epsilon^{2} f_{\alpha}^{(2)}+\epsilon^{3} f_{\alpha}^{(3)}+\mathcal{O}\left(\epsilon^{4}\right) \\
\partial_{t}=\epsilon \partial_{t}^{(1)}+\epsilon^{2} \partial_{t}^{(2)}+\epsilon^{3} \partial_{t}^{(3)}+\mathcal{O}\left(\epsilon^{4}\right) \\
\nabla=\epsilon \nabla^{(1)}
\end{gathered}
$$

The different moments of the EDF are given as:

$$
\begin{gathered}
\sum_{\alpha} f_{\alpha}^{(e q, 0)}=\rho \\
\sum_{\alpha} \mathbf{c}_{\alpha, j} f_{\alpha}^{(e q, 0)}=\rho \mathbf{u}_{j} \\
\mathbb{P}_{j k}^{(0)}=\sum_{\alpha} \mathbf{c}_{\alpha j} \mathbf{c}_{\alpha k} f_{\alpha}^{(e q, 0)}=\rho \mathbf{u}_{j} \mathbf{u}_{k}+c_{s}^{2} \rho \delta_{j k} \\
\mathbb{Q}_{j k l}^{(0)}=\sum_{\alpha} \mathbf{c}_{\alpha j} \mathbf{c}_{\alpha k} \mathbf{c}_{\alpha l} f_{\alpha}^{(e q, 0)}=c_{s}^{2} \rho\left(\mathbf{u}_{j} \delta_{k l}+\mathbf{u}_{k} \delta_{j l}+\mathbf{u}_{l} \delta_{j k}\right)
\end{gathered}
$$

Applying Eqs. A1 A3 and using a Taylor-McLaurin expansion on the LHS of Eq. 2, the following equations are recovered at different orders in $\epsilon$ :

$$
\begin{gathered}
\epsilon^{0}: f_{\alpha}^{(0)}=f_{\alpha}^{(e q, 0)} \\
\epsilon^{1}: D_{t}^{(1)} f_{\alpha}^{(0)}=-\frac{1}{\tau} f_{\alpha}^{(1)} \\
\epsilon^{2}: \partial_{t}^{(2)} f_{\alpha}^{(0)}+\tau_{2} D_{t}^{(1)^{2}} f_{\alpha}^{(0)}=-\frac{1}{\tau} f_{\alpha}^{(2)} \\
\epsilon^{3}: \partial_{t}^{(3)} f_{\alpha}^{(0)}+\tau_{2} \partial_{t}^{(2)} D_{t}^{(1)} f_{\alpha}^{(0)}+\tau_{2} D_{t}^{(1)} \partial_{t}^{(2)} f_{\alpha}^{(0)} \\
+\tau_{3} D_{t}^{(1)^{3}} f_{\alpha}^{(0)}=-\frac{1}{\tau} f_{\alpha}^{(3)}
\end{gathered}
$$


where $\tau_{2}=\frac{1}{2}-\tau$ and $\tau_{3}=\tau^{2}-\tau+\frac{1}{6}$, and $D_{t}^{(1)}=\partial_{t}^{(1)}+\nabla^{(1)}$. Given that at orders 1 and 2 in $\epsilon$, the conservation equations are readily available in the literature ${ }^{33}$, only the third-order equation will be treated in details below. The conservation equations are:

$$
\begin{gathered}
\epsilon^{1}: \partial_{t}^{(1)} \rho+\nabla^{(1)} \cdot \rho \mathbf{u}=0, \\
\epsilon^{1}: \partial_{t}^{(1)} \rho \mathbf{u}+\nabla^{(1)} \cdot \rho \mathbf{u} \otimes \mathbf{u}+\nabla^{(1)} \cdot c_{s}^{2} \rho \mathbb{I}=0, \\
\epsilon^{2}: \partial_{t}^{(2)} \rho=0, \\
\epsilon^{2}: \partial_{t}^{(2)} \rho \mathbf{u}+\tau_{2} \nabla^{(1)} \cdot \partial_{t}^{(1)} \mathbb{P}^{(0)}+\tau_{2} \nabla^{(1)} \cdot \nabla^{(1)} \cdot \mathbb{Q}^{(0)}=0,
\end{gathered}
$$

To obtain these moments, the following solvability conditions have been supposed to hold:

$$
\begin{aligned}
\sum_{\alpha} f_{\alpha}^{(i)} & =0, \forall i \neq 0 \\
\sum_{\alpha} \mathbf{c}_{\alpha} f_{\alpha}^{(i)} & =0, \forall i \neq 0
\end{aligned}
$$

The second and third terms on the LHS of Eq. A8 have been written separately since, according to Eqs. A6 and A7, operators $\partial_{t}^{(2)}$ and $D_{t}^{(1)}$ do not commute ${ }^{47}$. Taking the zerothorder moment of Eq. A8:

$$
\begin{aligned}
\partial_{t}^{(3)} \rho-\frac{1}{12} \nabla^{(1)} \otimes \nabla^{(1)}: \partial_{t}^{(1)} \mathbb{P}^{(0)} & \\
& -\frac{1}{12} \nabla^{(1)} \otimes \nabla^{(1)} \otimes \nabla^{(1)}: \mathbb{Q}^{(0)}=0 .
\end{aligned}
$$

The error term obtained at this scale agrees with results presented in $\frac{47 / 48}{}$. Taking the firstorder moment the following equation is recovered:

$$
\begin{aligned}
\partial_{t}^{(3)} \rho \mathbf{u}+\tau_{2} \nabla^{(1)} \partial_{t}^{(2)} \mathbb{P}^{(0)} & \\
+\left(\tau_{3}-\frac{1}{12}\right) \partial_{t}^{(1)} & \nabla^{(1)} \cdot\left(\partial_{t}^{(1)} \mathbb{P}^{(0)}+\nabla^{(1)} \cdot \mathbb{Q}^{(0)}\right) \\
& +\tau_{3} \nabla^{(1)} \cdot \nabla^{(1)} \cdot\left(\partial_{t}^{(1)} \mathbb{Q}^{(0)}+\nabla^{(1)} \cdot \mathbb{R}^{(0)}\right)=0 .
\end{aligned}
$$




\section{Appendix B: CE analysis of LKS at Euler and Navier-Stokes level}

To facilitate the expansion process, the equilibrium distribution function is written as:

$$
f_{\alpha}^{(e q)}=f_{\alpha}^{(e q, 0)}+\sum_{i=1}^{3} \epsilon^{i} f_{\alpha}^{(e q, i)}+\mathcal{O}\left(\epsilon^{4}\right),
$$

where:

$$
\begin{gathered}
f_{\alpha}^{(e q, 0)}=w_{\alpha} \rho\left[1+\frac{\mathbf{c}_{\alpha} \cdot \mathbf{u}}{c_{s}^{2}}+\frac{\mathbf{u} \otimes \mathbf{u}:\left(\mathbf{c}_{\alpha} \otimes \mathbf{c}_{\alpha}-c_{s}^{2} \mathbb{I}\right)}{2 c_{s}^{4}}\right], \\
f_{\alpha}^{(e q, i)}=w_{\alpha} \frac{A \mathbb{P}^{(i)}:\left(\mathbf{c}_{\alpha} \otimes \mathbf{c}_{\alpha}-c_{s}^{2} \mathbb{I}\right)}{2(A-\lambda) c_{s}^{4}}
\end{gathered}
$$

and:

$$
\mathbb{P}^{(i)}=\sum_{\alpha} \mathbf{c}_{\alpha} \otimes \mathbf{c}_{\alpha} f_{\alpha}^{(i)} .
$$

The different moments of these functions are given as:

$$
\begin{gathered}
\sum_{\alpha} f_{\alpha}^{(e q, i)}=0 \\
\sum_{\alpha} \mathbf{c}_{\alpha j} f_{\alpha}^{(e q, i)}=0 \\
\mathbb{P}_{j k}^{(e q, i)}=\sum_{\alpha} \mathbf{c}_{\alpha j} \mathbf{c}_{\alpha k} f_{\alpha}^{(e q, i)}=\frac{A}{A-\lambda} \mathbb{P}_{j k}^{(i)} \\
\mathbb{Q}_{j k l}^{(e q, i)}=\sum_{\alpha} \mathbf{c}_{\alpha j} \mathbf{c}_{\alpha k} \mathbf{c}_{\alpha l} f_{\alpha}^{(e q, i)}=0 \\
\mathbb{R}_{j k l m}^{(e q, i)}=\sum_{\alpha} \mathbf{c}_{\alpha j} \mathbf{c}_{\alpha k} \mathbf{c}_{\alpha l} \mathbf{c}_{\alpha m} f_{\alpha}^{(e q, i)}=\frac{A}{A-\lambda} \mathbb{R}_{j k l m}^{(i)}
\end{gathered}
$$

Applying Eqs. A1 A3, B1, B3 and using a Taylor-McLaurin expansion on the LHS of Eq. 7. the following equations are recovered at different orders in $\epsilon$ :

$$
\begin{gathered}
\epsilon^{0}: f_{\alpha}^{(0)}=f_{\alpha}^{(e q, 0)} \\
\epsilon^{1}: D_{t}^{(1)} f_{\alpha}^{(0)}=\frac{1}{\lambda}\left(f_{\alpha}^{(e q, 1)}-f_{\alpha}^{(1)}\right) \\
\epsilon^{2}: D_{t}^{(1)} f_{\alpha}^{(1)}+\partial_{t}^{(2)} f_{\alpha}^{(0)}+\frac{1}{2} D_{t}^{(1)^{2}} f_{\alpha}^{(0)}=\frac{1}{\lambda}\left(f_{\alpha}^{(e q, 2)}-f_{\alpha}^{(2)}\right)
\end{gathered}
$$

where $D_{t}^{(1)}=\partial_{t}^{(1)}+\mathbf{c}_{\alpha} \cdot \nabla^{(1)}$. Taking the zeroth-order moment of Eqs. B7 and B8.

$$
\partial_{t}^{(1)} \rho+\nabla^{(1)} \cdot \rho \mathbf{u}=0
$$




$$
\partial_{t}^{(2)} \rho=0
$$

Then, taking the first-order moment of Eq. B7.

$$
\epsilon^{1}: \partial_{t}^{(1)} \rho \mathbf{u}+\nabla^{(1)} \cdot \rho \mathbf{u} \otimes \mathbf{u}+\nabla^{(1)} \cdot \rho c_{s}^{2} \mathbb{I}=0
$$

At the scale of $\epsilon^{2}$, the moment of the following equation, obtained by multiplying Eq. B7 by $\mathbf{c}_{\alpha}$ has to be computed:

$$
\begin{aligned}
\epsilon^{2}: D_{t}^{(1)} \mathbf{c}_{\alpha} f_{\alpha}^{(1)}+\partial_{t}^{(2)} \mathbf{c}_{\alpha} f_{\alpha}^{(0)}+\frac{1}{2} D_{t}^{(1)^{2}} \mathbf{c}_{\alpha} f_{\alpha}^{(0)}= & \\
& \frac{1}{\lambda}\left(\mathbf{c}_{\alpha} f_{\alpha}^{(e q, 2)}-\mathbf{c}_{\alpha} f_{\alpha}^{(2)}\right)
\end{aligned}
$$

Multiplying Eq. B7 by $\frac{1}{2} D_{t}^{(1)} \mathbf{c}_{\alpha}$ :

$$
\frac{1}{2} D_{t}^{(1)^{2}} \mathbf{c}_{\alpha} f_{\alpha}^{(0)}=D_{t}^{(1)} \frac{1}{2 \lambda}\left(\mathbf{c}_{\alpha} f_{\alpha}^{(e q, 1)}-\mathbf{c}_{\alpha} f_{\alpha}^{(1)}\right)
$$

and putting it back into Eq. B12.

$$
\begin{aligned}
\epsilon^{2}: D_{t}^{(1)}\left(1-\frac{1}{2 \lambda}\right) \mathbf{c}_{\alpha} f_{\alpha}^{(1)}+\partial_{t}^{(2)} \mathbf{c}_{\alpha} f_{\alpha}^{(0)} & \\
& +D_{t}^{(1)} \frac{1}{2 \lambda} \mathbf{c}_{\alpha} f_{\alpha}^{(e q, 1)}=\frac{1}{\lambda}\left(\mathbf{c}_{\alpha} f_{\alpha}^{(e q, 2)}-\mathbf{c}_{\alpha} f_{\alpha}^{(2)}\right) .
\end{aligned}
$$

Taking the moment and using Eq. B5;

$$
\epsilon^{2}: \partial_{t}^{(2)} \rho \mathbf{u}+\nabla^{(1)} \cdot\left(1-\frac{1-\frac{A}{A-\lambda}}{2 \lambda}\right) \sum_{\alpha} \mathbf{c}_{\alpha} \otimes \mathbf{c}_{\alpha} f_{\alpha}^{(1)}=0 .
$$

Multiplying Eq. B12 by $\mathbf{c}_{\alpha} \otimes \mathbf{c}_{\alpha}$ and taking the moment:

$$
\left(1-\frac{A}{A-\lambda}\right) \sum_{\alpha} \mathbf{c}_{\alpha} \otimes \mathbf{c}_{\alpha} f_{\alpha}^{(1)}=-\lambda \partial_{t}^{(1)} \mathbb{P}^{(0)}-\lambda \nabla^{(1)} \cdot \mathbb{Q}^{(0)}
$$

Finally, plugging this result into Eq. B16 one gets the same macroscopic equation as the classical LBM, with the same leading-order error terms:

$$
\begin{aligned}
\epsilon^{2}: \partial_{t}^{(2)} \rho \mathbf{u}+\nabla^{(1)} \cdot\left(\frac{1}{2}+A-\lambda\right) \nabla^{(1)} \cdot \mathbb{Q}^{(0)} & \\
& +\nabla^{(1)} \cdot\left(\frac{1}{2}+A-\lambda\right) \partial_{t}^{(1)} \mathbb{P}^{(0)}=0
\end{aligned}
$$




\section{Appendix C: Higher-order CE analysis of LKS}

At order three in $\epsilon$ the following equation is obtained for LKS:

$$
\begin{aligned}
\epsilon^{3}: \partial_{t}^{(3)} f_{\alpha}^{(0)}+\lambda_{2} \partial_{t}^{(2)} D_{t}^{(1)} f_{\alpha}^{(0)}+ & \lambda_{2} D_{t}^{(1)} \partial_{t}^{(2)} f_{\alpha}^{(0)} \\
& +\partial_{t}^{(2)} f_{\alpha}^{(e q, 1)}+D_{t}^{(1)} f_{\alpha}^{(e q, 2)} \\
& +\lambda_{2} D_{t}^{(1)^{2}} f_{\alpha}^{(e q, 1)}+\lambda_{3} D_{t}^{(1)^{3}} f_{\alpha}^{(0)}=\frac{1}{\lambda}\left(f_{\alpha}^{(e q, 3)}-f_{\alpha}^{(3)}\right)
\end{aligned}
$$

Taking the zeroth-order moment of Eq. C1, the following mass conservation equation is found:

$$
\begin{aligned}
\partial_{t}^{(3)} \rho-\frac{1}{12} \nabla^{(1)} \otimes \nabla^{(1)}: \partial_{t}^{(1)} \mathbb{P}^{(0)} & \\
& -\frac{1}{12} \nabla^{(1)} \otimes \nabla^{(1)} \otimes \nabla^{(1)}: \mathbb{Q}^{(0)}=0 .
\end{aligned}
$$

Comparing this result to Eq. A14 it is clear that LKS does not affect the mass conservation equation up to third order in $\epsilon$. Multiplying Eq. C1 by $\mathbf{c}_{\alpha}$ :

$$
\begin{aligned}
& \epsilon^{3}: \partial_{t}^{(3)} \mathbf{c}_{\alpha} f_{\alpha}^{(0)}+\lambda_{2} \partial_{t}^{(2)} D_{t}^{(1)} \mathbf{c}_{\alpha} f_{\alpha}^{(0)}+\lambda_{2} D_{t}^{(1)} \partial_{t}^{(2)} \mathbf{c}_{\alpha} f_{\alpha}^{(0)} \\
&+\partial_{t}^{(2)} \mathbf{c}_{\alpha} f_{\alpha}^{(e q, 1)}+D_{t}^{(1)} \mathbf{c}_{\alpha} f_{\alpha}^{(e q, 2)}+\lambda_{2} D_{t}^{(1)^{2}} \mathbf{c}_{\alpha} f_{\alpha}^{(e q, 1)} \\
& \quad+\lambda_{3} D_{t}^{(1)^{3}} \mathbf{c}_{\alpha} f_{\alpha}^{(0)}=\frac{1}{\lambda}\left(\mathbf{c}_{\alpha} f_{\alpha}^{(e q, 3)}-\mathbf{c}_{\alpha} f_{\alpha}^{(3)}\right),
\end{aligned}
$$

where $\lambda_{2}=\frac{1}{2}-\lambda$ and $\lambda_{3}=\lambda^{2}-\lambda+\frac{1}{6}$. Now summing up over all discrete velocities and using Eqs. A4, B5 and B11;

$$
\begin{aligned}
\partial_{t}^{(3)} \rho \mathbf{u}+\lambda_{2} \partial_{t}(2) \overbrace{\left[\partial_{t}^{(1)} \rho \mathbf{u}+\nabla^{(1)} \cdot \mathbb{P}^{(0)}\right]}^{=0} & +\lambda_{2} \partial_{t}^{(1)} \partial_{t}^{(2)} \rho \mathbf{u} \\
& +\lambda_{2} \nabla^{(1)} \cdot \partial_{t}^{(2)} \mathbb{P}^{(0)}+\nabla^{(1)} \cdot \mathbb{P}^{(e q, 2)}
\end{aligned}
$$

Then, using Eqs. B5 for the second and third-order moments of $f_{\alpha}^{(e q, i)}$ :

$$
\begin{aligned}
\partial_{t}^{(3)} \rho \mathbf{u}+\lambda_{2} \partial_{t}^{(1)} \partial_{t}^{(2)} \rho \mathbf{u}+ & \lambda_{2} \nabla^{(1)} \cdot \partial_{t}^{(2)} \mathbb{P}^{(0)} \\
& +\frac{A}{A-\lambda} \nabla^{(1)} \cdot \mathbb{P}^{(2)}+\frac{2 \lambda_{2} A}{A-\lambda} \partial_{t}^{(1)} \nabla^{(1)} \cdot \mathbb{P}^{(1)} \\
& +\lambda_{3} \sum_{\alpha} D_{t}^{(1)^{3}} \mathbf{c}_{\alpha} f_{\alpha}^{(0)}=0
\end{aligned}
$$


The terms $\mathbb{P}^{(1)}$ and $\mathbb{P}^{(2)}$ can be evaluated as:

$$
\begin{gathered}
\mathbb{P}^{(1)}=(A-\lambda)\left[\partial_{t}^{(1)} \mathbb{P}^{(0)}+\nabla^{(1)} \cdot \mathbb{Q}^{(0)}\right] \\
\frac{\mathbb{P}^{(2)}}{A-\lambda}=\partial_{t}^{(2)} \mathbb{P}^{(0)}+\left(A-\lambda+\frac{1}{2}\right) \partial_{t}^{(1)^{2}} \mathbb{P}^{(0)} \\
+\left(A-\lambda+\frac{1}{2}+\frac{1}{2}-\lambda\right) \nabla^{(1)} \cdot \partial_{t}^{(1)} \mathbb{Q}^{(0)} \\
+\left(\frac{1}{2}-\lambda\right) \nabla^{(1)} \cdot \nabla^{(1)} \cdot \mathbb{R}^{(0)}
\end{gathered}
$$

Placing these expressions back into Eq. C5:

$$
\begin{aligned}
& \partial_{t}^{(3)} \rho \mathbf{u}+\left(\lambda_{2}+A\right) \nabla^{(1)} \cdot \partial_{t}^{(2)} \mathbb{P}^{(0)} \\
&+\left(\lambda_{3}+A \lambda_{2}-\frac{1}{12}\right) \partial_{t}^{(1)} \nabla^{(1)} \cdot\left(\partial_{t}^{(1)} \mathbb{P}^{(0)}+\nabla^{(1)} \cdot \mathbb{Q}^{(0)}\right) \\
& \quad+\left(\lambda_{3}+A \lambda_{2}\right) \nabla^{(1)} \cdot \nabla^{(1)} \cdot\left(\partial_{t}^{(1)} \mathbb{Q}^{(0)}+\nabla^{(1)} \cdot \mathbb{R}^{(0)}\right)=0 .
\end{aligned}
$$

It is readily observed that setting $A$ to zero and $\lambda=\tau$ Eq. C5 reduces to Eq. A15, recovering the classical SRT-LB model. Furthermore, developing the fourth-order equation it is seen that:

$$
\sum_{\alpha} \mathbf{c}_{\alpha} f_{\alpha}^{(4)}=\left(A+\lambda_{2}\right)\left(\lambda_{2}\left(A+\lambda_{2}\right)-\frac{1}{6}\right) f_{\alpha}^{(0)},
$$

Comparing the higher-order hydrodynamics limit presented here for the LKS, and those obtained for the SRT, it is seen that the modified collision operator results in different polynomial coefficients in front of higher-order moments, function of both physical viscosity and the free parameter in the LKS.

\section{Appendix D: CE analysis of bounce-back boundary condition}

For the sake of simplicity, and although readily extensible to more complex configurations, the case of a pressure-driven, steady Stokes flow in $x$-direction will be considered. The solid boundary is perpendicular to the fluid velocity vector. At a given boundary, for the boundary conditions to be $n$ th-order accurate -i.e., to be located exactly half-way between the last fluid node and its neighboring solid node with $n$ th-order accuracy, the following Taylor-Mclaurin expansion must hold:

$$
u_{0}=u_{x}(f)+\sum_{n} \frac{1}{n !}\left(\frac{\delta_{x}}{2}\right)^{n} \partial_{y}^{n} u_{x}(f)+\mathcal{O}\left(\delta_{x}^{n+1}\right) .
$$


Throughout the demonstration presented in this section, a Stokes flow is assumed, leading to negligible non-linear velocity terms. Furthermore for the sake of simplicity, $\delta_{x}$ is taken as 1. Hence, only linear velocity terms will be kept in the equilibrium distribution function. Within the context of the half-way bounce-back method, the incoming missing population is computed as:

$$
f_{\bar{\alpha}}=\left(1-\frac{1}{\lambda}\right) f_{\alpha}+\frac{1}{\lambda} f_{\alpha}^{(e q)}
$$

Using the multi-scale expansions of Eqs. A1 A3 and B1 B3 one can re-write the content of the bounce-back population as:

$$
\begin{aligned}
f_{\alpha}^{(0)}-f_{\bar{\alpha}}^{(0)}+\epsilon\left(f_{\alpha}^{(1)}-f_{\bar{\alpha}}^{(1)}\right)+\epsilon^{2}\left(f_{\alpha}^{(2)}-\right. & \left.f_{\bar{\alpha}}^{(2)}\right) \\
& +\frac{\epsilon}{\tau}\left(f_{\alpha}^{(e q, 1)}-f_{\alpha}^{(1)}\right)+\frac{\epsilon^{2}}{\tau}\left(f_{\alpha}^{(e q, 2)}-f_{\alpha}^{(2)}\right)=0 .
\end{aligned}
$$

Replacing $f_{\alpha}^{(1)}$ and $f_{\alpha}^{(2)}$ with:

$$
f_{\alpha}^{(1)}=f_{\alpha}^{(e q, 1)}-\lambda \mathbf{c}_{\alpha} \cdot \nabla^{(1)} f_{\alpha}^{(0)},
$$

and:

$$
\begin{aligned}
f_{\alpha}^{(2)}=f_{\alpha}^{(e q, 2)}-\lambda \mathbf{c}_{\alpha} \cdot \nabla^{(1)} f_{\alpha}^{(e q, 1)} & \\
& +\lambda\left(\lambda-\frac{1}{2}\right)\left(\mathbf{c}_{\alpha} \cdot \nabla^{(1)}\right)^{2} f_{\alpha}^{(0)},
\end{aligned}
$$

and using the following symmetry properties:

$$
\begin{gathered}
f_{\alpha}^{(0)}-f_{\bar{\alpha}}^{(0)}=2 w_{\alpha} \rho \frac{\mathbf{c}_{\alpha} \cdot \mathbf{u}}{c_{s}^{2}} \\
f_{\alpha}^{(0)}+f_{\bar{\alpha}}^{(0)}=2 w_{\alpha} \rho \\
f_{\alpha}^{(e q, 1)}=f_{\bar{\alpha}}^{(e q, 1)} \\
f_{\alpha}^{(e q, 2)}=f_{\bar{\alpha}}^{(e q, 2)}
\end{gathered}
$$

the following equation is obtained:

$$
\begin{aligned}
c_{\alpha x} u_{x}+\frac{1}{2} c_{\alpha y} \partial_{y}^{(1)} c_{\alpha x} u_{x} & +\left(\lambda-\frac{1}{2}\right)^{2}\left(c_{\alpha y} \partial_{y}^{(1)}\right)^{2} c_{\alpha x} u_{x} \\
& -\left(\lambda-\frac{1}{2}\right)\left[\frac{c_{\alpha x} \partial_{x}^{(1)} c_{s}^{2} \rho}{\rho}+\frac{1}{2} \frac{\left(c_{\alpha x} \partial_{x}^{(1)}\right)^{2} c_{s}^{2} \rho}{\rho}\right] \\
& -\left(\lambda-\frac{1}{2}\right) \frac{\mathbf{c}_{\alpha} \cdot \nabla^{(1)} c_{s}^{2} f_{\alpha}^{(e q, 1)}}{w_{\alpha} \rho}=0 .
\end{aligned}
$$


where $c_{\alpha x}$ and $c_{\alpha y}$ are the $x$ - and $y$-components of $\mathbf{c}_{\alpha}$. The terms $c_{\alpha x} \partial_{x} c_{s}^{2} \rho$ and $\left(\mathbf{c}_{\alpha x} \partial_{x}\right)^{2} c_{s}^{2} \rho$ can be evaluated using Eqs. B17 for a steady pressure-driven Stokes flow as:

$$
\begin{gathered}
\partial_{x} \rho c_{s}^{2}=-c_{s}^{2} \rho\left(\frac{1}{2}+A-\lambda\right) \partial_{y}^{2} u_{x}, \\
\partial_{x}^{2} c_{s}^{2} \rho=0 .
\end{gathered}
$$

Plugging these expressions back into D7 we get:

$$
\begin{aligned}
& c_{\alpha x} u_{x}+\frac{1}{2} c_{\alpha y} \partial_{y}^{(1)} c_{\alpha x} u_{x} \\
& +\left(\lambda-\frac{1}{2}\right)\left(\lambda-A-\frac{1}{2}\right)\left(1-c_{s}^{2}\right)\left(c_{\alpha y} \partial_{y}^{(1)}\right)^{2} c_{\alpha x} u_{x}=0,
\end{aligned}
$$

while a similar analysis for the SRT model would lead to:

$$
\begin{aligned}
& c_{\alpha x} u_{x}+\frac{1}{2} c_{\alpha y} \partial_{y}^{(1)} c_{\alpha x} u_{x} \\
& +\left(\tau-\frac{1}{2}\right)^{2}\left(1-c_{s}^{2}\right)\left(c_{\alpha y} \partial_{y}^{(1)}\right)^{2} c_{\alpha x} u_{x}=0 .
\end{aligned}
$$

